\title{
Taxifolin inhibits amyloid- $\beta$ oligomer formation and fully restores vascular integrity and memory in cerebral amyloid angiopathy
}

Satoshi Saito ${ }^{1,2^{*}}$, Yumi Yamamoto ${ }^{1}$, Takakuni Maki ${ }^{2}$, Yorito Hattori ${ }^{3}$, Hideki Ito ${ }^{4}$, Katsuhiko Mizuno ${ }^{5}$, Mariko Harada-Shiba ${ }^{1,6}$, Raj N. Kalaria ${ }^{7}$, Masanori Fukushima ${ }^{8}$, Ryosuke Takahashi ${ }^{2}$ and Masafumi Ihara ${ }^{3^{*}}$

\begin{abstract}
Cerebral amyloid angiopathy (CAA) induces various forms of cerebral infarcts and hemorrhages from vascular amyloid$\beta$ accumulation, resulting in acceleration of cognitive impairment, which is currently untreatable. Soluble amyloid- $\beta$ protein likely impairs cerebrovascular integrity as well as cognitive function in early stage Alzheimer's disease. Taxifolin, a flavonol with strong anti-oxidative and anti-glycation activities, has been reported to disassemble amyloid- $\beta$ in vitro but the in vivo relevance remains unknown. Here, we investigated whether taxifolin has therapeutic potential in attenuating CAA, hypothesizing that inhibiting amyloid- $\beta$ assembly may facilitate its clearance through several elimination pathways. Vehicle- or taxifolin-treated Tg-SwDI mice (commonly used to model CAA) were used in this investigation. Cognitive and cerebrovascular function, as well as the solubility and oligomerization of brain amyloid- $\beta$ proteins, were investigated. Spatial reference memory was assessed by water maze test. Cerebral blood flow was measured with laser speckle flowmetry and cerebrovascular reactivity evaluated by monitoring cerebral blood flow changes in response to hypercapnia. Significantly reduced cerebrovascular pan-amyloid- $\beta$ and amyloid- $\beta_{1-40}$ accumulation was found in taxifolin-treated Tg-SwDI mice compared to vehicle-treated counterparts $(n=5)$. Spatial reference memory was severely impaired in vehicle-treated Tg-SwDI mice but normalized after taxifolin treatment, with scoring similar to wild type mice $(n=10-17)$. Furthermore, taxifolin completely restored decreased cerebral blood flow and cerebrovascular reactivity in Tg-SwDI mice $(n=4-6)$. An in vitro thioflavin-T assay showed taxifolin treatment resulted in efficient inhibition of amyloid- $\beta_{1-40}$ assembly. In addition, a filter trap assay and ELISA showed Tg-SwDI mouse brain homogenates exhibited significantly reduced levels of amyloid- $\beta$ oligomers in vivo after taxifolin treatment $(n=4-5)$, suggesting the effects of taxifolin on CAA are attributable to the inhibition of amyloid- $\beta$ oligomer formation. In conclusion, taxifolin prevents amyloid- $\beta$ oligomer assembly and fully sustains cognitive and cerebrovascular function in a CAA model mice. Taxifolin thus appears a promising therapeutic approach for CAA.
\end{abstract}

Keywords: Alzheimer's disease, Cerebral amyloid angiopathy, Oligomer, Taxifolin, Treatment

\footnotetext{
*Correspondence: saitosa@ncvc.go.jp; ihara@ncvc.go.jp

${ }^{1}$ Department of Regenerative Medicine and Tissue Engineering, National

Cerebral and Cardiovascular Center, 5-7-1 Fujishiro-dai, Suita, Osaka 565-8565,

Japan

${ }^{3}$ Department of Stroke and Cerebrovascular Diseases, National Cerebral and

Cardiovascular Center, 5-7-1 Fujishiro-dai, Suita, Osaka 565-8565, Japan

Full list of author information is available at the end of the article
} 


\section{Introduction}

Cerebral amyloid angiopathy (CAA) is pathologically characterized by the deposition of amyloid- $\beta$ within small cerebral vessels. CAA is a major cause of lobar intracerebral hemorrhage, cerebral infarction and cognitive impairment in the elderly, though there are currently no established treatments [18, 65, 69].

Amyloid- $\beta$ deposition within cerebral capillaries has been consistently associated with the apolipoprotein $\mathrm{E}$ $\varepsilon 4$ allele and is frequently concomitant with Alzheimer's disease [66]. Accumulating lines of evidence have shown CAA plays a pivotal role in the pathogenesis of dementia [36, 47]. Structural network alterations and neurological dysfunction have been reported in CAA patients $[10,55]$. CAA has been associated with Alzheimer's disease in community-based older persons [8]. Recent pathological reports have shown that CAA is extremely common in sporadic Alzheimer's disease [35], suggesting a strong, bidirectional relationship. Some forms of familial Alzheimer's disease and Down syndrome also frequently exhibit CAA $[56,74]$. CAA is commonly regarded as a pathological hallmark of Alzheimer's disease, in parallel to senile plaque and neurofibrillary tangle deposition $[17,25]$.

Inhibiting amyloid- $\beta$ assembly is a potential approach in the prevention of progression of both Alzheimer's disease and CAA as soluble amyloid- $\beta$ is likely to impair cerebrovascular integrity as well as cognitive function in the early stages of Alzheimer's disease [21-23]. Cerebrovascular amyloidosis reduces the ability of vessels to constrict and dilate in response to physiologic stimulation, whereas impaired vasoreactivity predisposes ischemic damage $[16,20]$. In addition to non-soluble forms, soluble amyloid- $\beta$ deposition also induces cerebrovascular dysfunction, which is known to start before the appearance of visible amyloid- $\beta$ deposits [22].

Silymarin, an extract of Silybum marianum, has been identified as a candidate in amyloid- $\beta$ assembly inhibition. Silymarin has been reported to reduce amyloid- $\beta$ plaque pathology in Alzheimer's disease model mice [48]. However, no improvement in cognitive and cerebrovascular function was reported in subsequent experimental studies, potentially as silymarin is a mixture of several compounds, meaning its essential effector component remains obscure. Recently, taxifolin has been found to be the active component of silymarin, preventing amyloid- $\beta$ aggregation in vitro [60]. Taxifolin, also known as a dihydroquercetin, is a catechol-type flavonoid with strong anti-oxidant and anti-glycation activities $[19,26]$. We therefore hypothesized taxifolin would more effectively ameliorate cerebrovascular dysfunction due to CAA, rather than the senile plaques in Alzheimer's disease, as only a small amount of its metabolites have been detected in the rat brain parenchyma after administration, suggesting low permeability across the blood-brain barrier (BBB) [76]. Here, we investigated whether taxifolin would modify the burden of CAA. Vascular amyloid- $\beta$ accumulation is mainly composed of amyloid- $\beta_{1-40}$, while senile plaques are characterized by amyloid- $\beta_{1-42}$ accumulation. The pathogenicity of amyloid- $\beta_{1-40}$ in CAA has been established in many previous experiments $[49,50,53]$. The purpose of this study was therefore to examine the potential protective effects of taxifolin on amyloid- $\beta_{1-40}$ metabolism and cerebrovascular dysfunction in CAA model mice. This experimental approach may provide a rationale for subsequent clinical trials examining the efficacy of taxifolin in patients with CAA.

\section{Materials and methods}

\section{Animals}

We obtained heterozygous C57BL/6 J-Tg(Thy1-APPSwDutIowa)BWevn/J, also known as the Tg-SwDI, mice from Jackson Laboratory, Bar Harbor, USA. Low levels of the human APP gene with Swedish/Dutch/Iowa triple mutations are expressed in neurons under the control of the mouse Thy1 promoter on a pure C57BL/6 J mice background [12]. Homozygous Tg-SwDI were generated from heterozygous Tg-SwDI mice and verified by backcrossing. Male homozygous Tg-SwDI and wild type (WT) mice aged 8 to 14 months (weighing 30-50 g) were examined in the present study. C57BL/6 J and C57BL/6 N mice were obtained from the Japan SLC, Hamamatsu, Japan. C57BL/6 J mice were used as WT controls, and C57BL/6 N mice aged 8 to 9 weeks were used for pharmacokinetic analysis only. Experimental mice were randomly assigned to taxifolin versus vehicle group and fed with pelleted chow containing 3\% taxifolin (Ametis JSC, Blagoveshchensk, Russia) or standard pelleted chow only from the age of 1 month until death, unless stated otherwise. All mice were housed in a room with a 12-h light/dark cycle (lights on at 7:00 a.m.), with access to food and water ad libitum. No more than five mice were housed per cage, and were separated when fighting was noted. All study protocols were performed respecting animal dignity and were applicable to international and Japanese guidelines for the care, and the ethical standards of Kyoto University (Permit Number: MedKyo15274), and National Cerebral and Cardiovascular Center (Permit Number: 16068).

\section{Pharmacokinetic analysis}

In the first cohort, male WT mice aged 8 weeks were given taxifolin solution once at $30,100,300 \mathrm{mg} / \mathrm{kg}$ of body weight by gavage into the stomach using a bluntended needle, followed by blood collection, via the vena cava, at $0.25,0.5,1.0,2.0,4.0$, and $8.0 \mathrm{~h}$ after dosing. In the second cohort, mice aged 9 weeks were fed with pelleted chow containing either $1 \%$ or $3 \%$ taxifolin for 
5 days. Blood was collected via the vena cava at 8:00, 13:00, 18:00, 23:00, and 3:00. Blood samples were collected with heparinized capillary tubes to prepare plasma, then allowed to clot for $30 \mathrm{~min}$ at room temperature before centrifugation for $10 \mathrm{~min}$ at $3000 \times \mathrm{g}$ to collect serum.

Levels of taxifolin in brain homogenates extracted from WT and Tg-SwDI mice were also investigated. The brains of Tg-SwDI mice aged 6 and 14 months were collected at 10:00 and 13:00, respectively. Tg-SwDI mice were fed with pelleted chow containing 3\% taxifolin from the age of 1 month. WT and Tg-SwDI mice were deeply anesthetized by isoflurane inhalation and transcardially perfused with saline. Brains were harvested in saline and the homogenized lysates used for evaluation of taxifolin concentration. Taxifolin concentrations were measured using liquid chromatography/mass spectrometry/mass spectrometry. The limits of quantification in blood and brain were $3-10 \mathrm{ng} / \mathrm{mL}(9.9-33 \mathrm{nM})$ and $15-$ $30 \mathrm{ng} / \mathrm{g}$, respectively.

\section{Morris water maze test}

Spatial reference memory was assessed using the Morris water maze test, as described earlier [27, 28]. A circular pool (diameter, $120 \mathrm{~cm}$; depth, $40 \mathrm{~cm}$ ) and a set of video analysis systems (EthoVision XT5; Noldus, Wageningen, Netherlands) were used. The pool was filled with water containing non-toxic white paint to a depth of $11 \mathrm{~cm}$. A clear, circular platform (diameter, $10 \mathrm{~cm}$ ) was submerged $1 \mathrm{~cm}$ below the water surface in the center of one quadrant of the pool (target quadrant). A red 'cross' sign and a blue 'upward arrow' (placed oppositely) were used as orientation cues to the swimming pool for the mice.

On the first 4 days, four trials per day were performed with a 30-minute interval between attempts (acquisition phase). The platform was kept in the same position during the acquisition phase. Mice were placed at the starting position (the quadrant adjacent to the target) and released into the water. Each mouse was allowed to swim for $60 \mathrm{~s}$, discover the hidden platform, and climb onto it. The trial was immediately terminated after the mouse arrived on the platform or after $60 \mathrm{~s}$ had elapsed. If a mouse succeeded in climbing onto the platform, it was permitted to remain for $10 \mathrm{~s}$. If a mouse did not reach the platform within $60 \mathrm{~s}$, it was placed on the platform and allowed to remain for $15 \mathrm{~s}$. Escape latency (time to goal) and total swimming distance to reach the platform were recorded.

On the fifth day, mice were subjected to a probe trial session where the platform was removed from the pool and mice allowed to swim for $60 \mathrm{~s}$ to search for it. The time spent in the platform quadrant and the number of entries into the target quadrant was recorded.

\section{Measurement of cerebral blood flow}

Relative cerebral blood flow (CBF) of WT and Tg-SwDI mice was recorded using laser speckle flowmetry (Omegazone-2, Omegawave, Fuchu, Japan), as previously reported but with modifications [30, 42]. Laser speckle flowmetry obtains high-resolution, two-dimensional imaging and has a linear relationship with absolute $\mathrm{CBF}$ values [4]. Anesthesia was induced with $2 \%$, and maintained with $1.5 \%$, isoflurane in $80 \%$ nitrous oxide and $20 \%$ oxygen. An anesthesia mask for mice was used for isoflurane inhalation without tracheal intubation. The scalp was removed by a midline incision to expose the skull throughout CBF evaluation. CBF was measured in identically-sized regions of interest (circle $1 \mathrm{~mm}$ in diameter), located $1 \mathrm{~mm}$ posterior and $2 \mathrm{~mm}$ lateral from the bregma, corresponding to regions around Heubner's anastomoses, connecting the dorsal branches of the anterior cerebral artery and the middle cerebral artery. Average CBF values in the bilateral hemispheres were recorded.

\section{Evaluation of vascular responses to hypercapnia}

In order to examine cerebrovascular reactivity (CVR), the CBF response to hypercapnia was evaluated in WT and Tg-SwDI mice, with minor modifications to the methods described previously [29]. Mice were anesthetized with an intraperitoneal injection of $\alpha$-chloralose $(50 \mathrm{mg} / \mathrm{kg})$ and urethane $(750 \mathrm{mg} / \mathrm{kg})$. The stability of anesthesia level was checked by testing corneal reflexes and motor responses to tail pinch. The trachea was intubated and mice mechanically ventilated at a stroke volume of $5 \mathrm{~mL} / \mathrm{kg}$ body weight and ventilation rate of 100 strokes/minute with a ventilator. CBF was monitored by laser speckle flowmetry. To induce hypercapnia, mice were ventilated with $5 \%$ carbon dioxide for $5 \mathrm{~min}$, followed by ventilation with $20 \%$ oxygen containing air. After measurement of baseline CBF, changes in response to hypercapnia were monitored for $5 \mathrm{~min}$, with values obtained every $1 \mathrm{~min}$.

\section{Histologic investigation}

WT and Tg-SwDI mice were deeply anesthetized by an intraperitoneal injection of sodium pentobarbital $(40 \mathrm{mg} / \mathrm{kg})$ and transcardially perfused with $0.01 \mathrm{M}$ phosphate buffered saline, followed by $4 \%$ paraformaldehyde in $0.1 \mathrm{M}$ phosphate buffer. The removed brains were post-fixed in $4 \%$ paraformaldehyde overnight and embedded in paraffin, then sliced into $6 \mu \mathrm{m}$-thick sagittal sections $1 \mathrm{~mm}$ lateral from the midline. For thioflavin-S staining, sections were deparaffinized and immersed in a $100 \mu \mathrm{M}$ thioflavin-S solution containing $50 \%$ ethanol for $30 \mathrm{~min}$, then washed in $100 \%$ ethanol for $1 \mathrm{~min}$. The fluorescent images were captured with a digital camera (BZ-9000, Keyence, Osaka, Japan). For Perls-Stieda's iron staining, sections were 
immersed in a solution of an equal amount of hydrochloric acid and potassium ferrocyanide for $30 \mathrm{~min}$, followed by counterstaining with $0.1 \%$ nuclear fast red for $3 \mathrm{~min}$.

For immunohistochemistry, mouse anti-amyloid- $\beta_{1-16}$ antibody (diluted 1:500, 6E10; BioLegend, San Diego, USA), rabbit anti-amyloid- $\beta_{1-40}$ (diluted 1:500, FCA3340, Merck Millipore, Darmstadt, Germany), rabbit antiamyloid- $\beta_{1-42}$ (diluted 1:500, FCA3542, Merck Millipore) and rabbit anti-RAGE (receptor for advanced glycation end-products, receptor for AGEs) (diluted 1:100, ab3611, Abcam, Cambridge, UK) antibodies were used as primary antibodies after formic acid or heat-mediated antigen retrieval. The sections were subsequently treated with labeled polymer, prepared by combining amino acid polymers with peroxidase and secondary antibody, which is reduced to Fab' fragment (Nichirei Biosciences, Tokyo, Japan). Sections were rinsed with phosphate buffered saline for $15 \mathrm{~min}$ between each step and finally visualized with $0.01 \%$ diaminobenzidine tetrahydrochloride and $0.005 \% \mathrm{H}_{2} \mathrm{O}_{2}$ in $50 \mathrm{mM}$ Tris- $\mathrm{HCl}(\mathrm{pH}$ 7.6).

Densitometric analysis of amyloid- $\beta$ accumulation was performed blindly to animal groups by setting regions of interest in the whole hippocampus in the immunostained sections. The percentage area of amyloid- $\beta$ positive regions with the identical threshold was calculated using the Image-J software package (National Institutes of Health, Bethesda, USA).

The degree of RAGE expression in leptomeningeal and cortical arteries was classified into four grades: "none" (no expression), "low" (focal expression in less than $50 \%$ of whole circumference of vascular wall), "moderate" (intermediate expression in the vascular wall), and "high" (robust expression surrounding in more than $80 \%$ of the entire vascular wall). RAGE expression score was calculated from the average of qualified grading of RAGE expression $(0=$ none, 1 = low, $2=$ moderate, $3=$ high). Fifty leptomeningeal or cortical arteries (five per mouse) were analyzed in randomly selected regions in five vehicle-treated and five taxifolin-treated $\mathrm{Tg}$-SwDI mice.

\section{Immunofluorescence for the evaluation of vascular amyloid- $\beta$ accumulation}

$\mathrm{Tg}$-SwDI mice were deeply anesthetized with an intraperitoneal injection of sodium pentobarbital $(40 \mathrm{mg} / \mathrm{kg})$, then perfused transcardially with $0.01 \mathrm{M}$ phosphate buffered saline, followed by $60 \mathrm{mg}$ of fluorescein isothiocyanate (FITC) dextran in $0.9 \%$ saline, then $4 \%$ paraformaldehyde in $0.1 \mathrm{M}$ phosphate buffer. Brains were removed and post-fixed in $4 \%$ paraformaldehyde for $12 \mathrm{~h}$, then snap-frozen and sliced into $20 \mu \mathrm{m}$-thick sagittal sections at $1 \mathrm{~mm}$ lateral from the midline. Sections were then incubated at $4{ }^{\circ} \mathrm{C}$ overnight with a mouse anti-amyloid- $\beta_{1-16}$ primary antibody (diluted 1:500), followed by fluorescent dye conjugated secondary antibody at room temperature for $60 \mathrm{~min}$ (diluted 1:200, Thermo Fisher Scientific, Waltham, USA).

\section{Thioflavin-T fluorescence assay}

A SensoLyte Thioflavin T $\beta$-Amyloid (1-40) Aggregation Kit was used for the in vitro amyloid- $\beta$ aggregation assay (AnaSpec, Fremont, USA). Thioflavin-T fluorescence assay measures change in the intensity of fluorescence emitted when amyloid fibrils or oligomers are bound to thioflavin$\mathrm{T}[32,33,41]$. Samples including $50 \mu \mathrm{M}$ amyloid- $\beta_{1-40}$ and thioflavin- $\mathrm{T}$ were incubated at $37^{\circ} \mathrm{C}$. Fluorescence intensity of each sample was recorded every $10 \mathrm{~min}$ for $6 \mathrm{~h}$ using a Wallac 1420 Multilabel counter (Perkin Elmer, Waltham, USA) with 440/486 nm excitation/emission filters set. Each sample was triply prepared and the mean fluorescence expressed in relative fluorescence units.

\section{Transmission electron microscopy (TEM)}

The amyloid- $\beta_{1-40}$ aggregates produced in the thioflavin$\mathrm{T}$ assay were examined by electron microscope [43]. After 6-hour incubation at $37{ }^{\circ} \mathrm{C}$, the samples were centrifuged at $16,000 \times \mathrm{g}$ at $4{ }^{\circ} \mathrm{C}$ for $90 \mathrm{~min}$. The resultant pellets were suspended in distilled water and applied to a carbon film with 400 copper grids. The samples were negatively stained with $2 \%$ uranyl acetate for 2 min. Formation of fibrils was examined by electron microscopy (JEM-1200EX, JEOL, Akishima, Japan).

\section{Filter trap assay}

$\mathrm{Tg}$-SwDI mice were deeply anesthetized with an intraperitoneal injection of sodium pentobarbital $(40 \mathrm{mg} / \mathrm{kg})$ and transcardially-perfused with $0.01 \mathrm{M}$ phosphate buffered saline. Whole frozen cerebrum were homogenized and harvested in Tris buffered saline (TBS, pH 7.5) and protease inhibitors cocktail (Nacalai Tesque, Kyoto, Japan). Soluble, extracellular-enriched proteins were collected from homogenized lysates after centrifugation at $100,000 \times \mathrm{g}$ at $4{ }^{\circ} \mathrm{C}$ for $60 \mathrm{~min}$. The precipitate was again added with TBS and protease inhibitors, followed by centrifugation at $100,000 \times \mathrm{g}$ at $4{ }^{\circ} \mathrm{C}$ for $60 \mathrm{~min}$. The precipitate was added with $70 \%$ formic acid and centrifuged at $100,000 \times \mathrm{g}$ at $4{ }^{\circ} \mathrm{C}$ for $60 \mathrm{~min}$. The resultant supernatant was neutralized with $1 \mathrm{M}$ Tris buffer $(\mathrm{pH} 11.0)$, which was used as the TBS insoluble fraction.

Filter trap assay was conducted, as described previously [40, 75]. Briefly, the protein concentration of the samples in TBS soluble fraction was measured and an equal amount of protein was subjected to vacuum filtration through a 96-well dot blot apparatus (Bio-Rad Laboratories, Hercules, USA) containing $200 \mathrm{~nm}$ poresized mixed cellulose ester membranes. The resultant membranes were then blocked by TBS containing $4 \%$ 
skim milk and incubated with primary antibody at $4{ }^{\circ} \mathrm{C}$ overnight. Mouse anti-amyloid- $\beta_{1-16}$ antibody (diluted $1: 1000,6 E 10)$ and rabbit anti-amyloid- $\beta$ oligomer antibody (diluted 1:1000, A11; Thermo Fisher Scientific, Waltham, USA) was used. The signal was visualized using horseradish peroxidase-conjugated anti-mouse and anti-rabbit IgG secondary antibodies (diluted 1:2000, Jackson Immuno Research Laboratories, West Grove, USA) with enhanced chemiluminescence (Merck Millipore). Immunoblot membranes were developed using the LAS4000 Imaging System (Fujifilm, Tokyo, Japan). Densitometric measurement was performed using Image-J. Duplicate samples from each mouse were prepared for each primary antibody and the mean value measured.

\section{Enzyme-linked immunosorbent assay (ELISA)}

The concentration of amyloid- $\beta_{1-40}$, amyloid- $\beta_{1-42}$ and amyloid- $\beta$ oligomers in the TBS soluble fraction extracted from whole cerebrum of $\mathrm{Tg}$-SwDI mice was estimated by ELISA [39]. The levels of amyloid- $\beta_{1-40}$ and amyloid- $\beta_{1-42}$ were also examined in the TBS insoluble fraction. The concentration of amyloid- $\beta_{1-40}$, amyloid- $\beta_{1-}$ 42 and amyloid- $\beta$ oligomer (82E1-specific) was measured, according to the manufacture's protocol (Human/Rat $\beta$ Amyloid (40) ELISA Kit Wako II, Wako, Osaka, Japan; Human/Rat $\beta$ Amyloid (42) ELISA Kit Wako, High-Sensitive; Human Amyloid $\beta$ Oligomers Assay Kit, Immuno-Biological Laboratories, Fujioka, Japan). Blood levels of amyloid- $\beta_{1-40}$ and amyloid- $\beta_{1-42}$ were also examined. Blood samples were obtained from the vena cava under isoflurane anesthesia, which were collected with heparinized capillary tubes to prepare plasma. The samples were allowed to clot for $30 \mathrm{~min}$ at room temperature followed by centrifugation for $5 \mathrm{~min}$ at $6000 \times \mathrm{g}$ to collect serum.

\section{Statistical analysis}

All values were expressed as mean \pm SD unless stated otherwise. Statistical analysis was conducted using Student's $t$ test or ANOVA followed by post hoc Turkey or Games-Howell tests. Differences with $p<0.05$ were considered statistically significant in all analyses. Statistical analysis was performed using SPSS Statistics version 23.0 (IBM, Armonk, USA).

\section{Results}

\section{Pharmacokinetics and safety profile of taxifolin}

We firstly investigated the pharmacokinetic profile of taxifolin in 8-week-old WT mice given by solution orally via gavage. Blood levels of taxifolin were increased in a dose-dependent manner (Fig. 1a). In $300 \mathrm{mg} / \mathrm{kg}$ taxifolin-treated mice, maximum concentration (Cmax) and elimination half-life period were recorded as $18.87 \mu \mathrm{M}(5741 \mathrm{ng} / \mathrm{mL})$ and $0.67 \mathrm{~h}$, respectively, as in previous rat studies [70].
We evaluated changes in taxifolin concentrations in 9week-old WT mice receiving each type of pelleted chow: $1 \%$ or $3 \%$ taxifolin-containing food. Although a previous investigation suggested more than $10 \mu \mathrm{M}$ of taxifolin was needed to disassemble amyloid- $\beta$ in vitro [61, 72], the Cmax of $1 \%$ taxifolin-treated mice was $0.199 \mu \mathrm{M}$ $(60.52 \mathrm{ng} / \mathrm{mL})$, meaning $1 \%$ taxifolin would not be sufficient to achieve amyloid- $\beta$ disaggregation in vivo. In contrast, blood taxifolin levels in 3\% taxifolin-treated WT mice were substantially elevated and Cmax recorded as $7.53 \mu \mathrm{M}(2290 \mathrm{ng} / \mathrm{mL})$ (Fig. 1b). Blood taxifolin levels in Tg-SwDI mice receiving 3\% taxifolin treatments were almost equivalent to those in $3 \%$ taxifolin-treated WT mice (data not shown); we therefore administered pelleted chow containing 3\% taxifolin in subsequent experiments. We also estimated taxifolin levels in brain homogenates extracted from WT and Tg-SwDI mice treated with 3\% taxifolin (Fig. 1b and c). The estimates suggested the dose of taxifolin was too low to reach a sufficient level in the brain parenchyma.

The safety profile of taxifolin has been studied previously $[7,79]$. Consistent with these reports, animal survival rates were comparable among vehicle- or taxifolin-treated WT and Tg-SwDI mice groups (Fig. 1d). Taxifolin treatment did not induce any apparent systemic abnormalities in $\mathrm{Tg}$ SwDI mice.

\section{Complete normalization of spatial reference memory impairment in taxifolin-treated Tg-SwDI mice}

We examined whether taxifolin treatment affected spatial learning and reference memory impairment by performing the Morris water maze test in 8-month-old WT and Tg-SwDI mice. During the acquisition phase, compared with WT, vehicle-treated Tg-SwDI mice exhibited significantly longer escape latencies suggesting severely impaired reference memory. However, the latency in taxifolin-treated Tg-SwDI mice was comparable to WT mice (Fig. 2a). Recorded total swimming distance did not differ among WT, vehicle-treated Tg-SwDI, and taxifolin-treated $\mathrm{Tg}$-SwDI, mice (Fig. 2b). In the probe trial phase, the time present in the platform quadrant and the number of entries into the target quadrant in WT and taxifolin-treated Tg-SwDI mice was comparable and both values were significantly greater than those in vehicle-treated $\mathrm{Tg}$-SwDI mice (Fig. $2 \mathrm{c}$ and $\mathrm{d}$ ), indicating taxifolin completely normalized spatial reference memory impairment in Tg-SwDI mice.

\section{Restoration of CBF and CVR in taxifolin-treated Tg-SwDI mice}

Impairments in cerebral circulation and vascular reactivity have substantial roles in the onset and progression of cognitive dysfunction in patients with CAA and several animal models $[16,42]$. We firstly investigated resting 


\section{a}

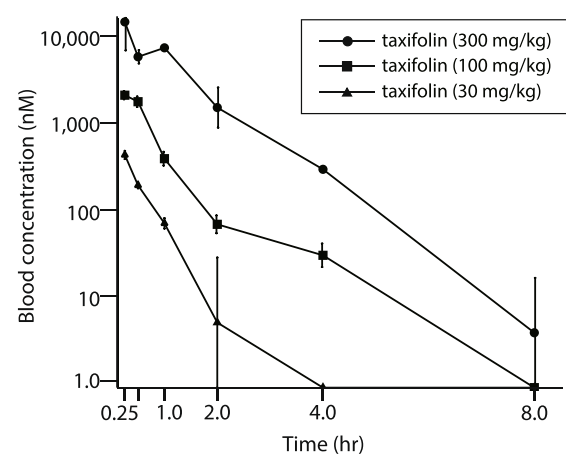

C

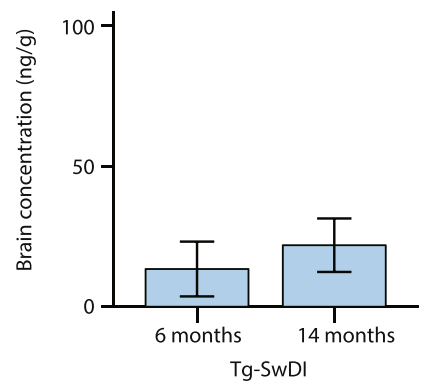

b

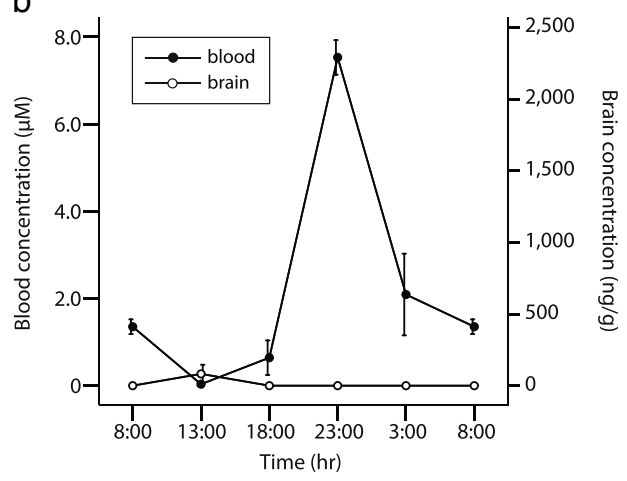

d

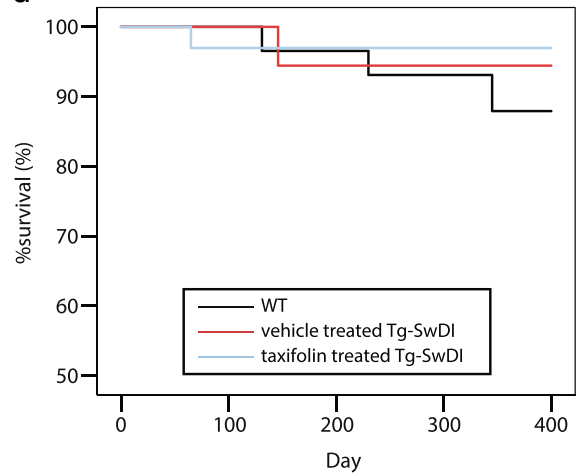

Fig. 1 Pharmacokinetics and safety profile of taxifolin. Taxifolin is mostly degraded within an hour, and is rarely detected in the brain parenchyma. Survival rate is not affected by taxifolin treatment. a Line graphs showing changes in blood taxifolin concentration in 8-week-old WT mice receiving $30 \mathrm{mg} / \mathrm{kg}$ (filled triangle), $100 \mathrm{mg} / \mathrm{kg}$ (filled square), or $300 \mathrm{mg} / \mathrm{kg}$ (filled circle) of single taxifolin administration per oral ( $n=3$ for each). $\mathbf{b}$ Line graphs showing blood and brain taxifolin concentration in 9-week-old WT mice fed with the pelleted chow containing 3\% taxifolin $(n=3)$. The first vertical axis represents blood concentration (filled circle). The second axis represents brain concentration (open circle). c A histogram showing brain taxifolin concentration in Tg-SwDI mice with taxifolin treatment at the age of 6 and 14 months ( $n=5-6$ for each groups). d Kaplan-Meier plots showing taxifolin does not affect survival rate of WT (black line) and Tg-SwDI mice treated with vehicle (red line) or taxifolin (blue line) ( $n=28-33$ for each group). Error bars indicate $\pm \mathrm{SE}$

CBF of WT and Tg-SwDI mice at 12 months of age using laser speckle flowmetry. Vehicle-treated $\mathrm{Tg}$-SwDI mice exhibited significantly decreased CBF compared with WT mice (Fig. 3a; left and middle panels). However, taxifolin treatment restored CBF reduction in Tg-SwDI mice (Fig. 3a; right panel). Resting CBF of taxifolin-treated $\mathrm{Tg}$-SwDI mice was comparable to that of WT mice (Fig. 3b).

We then evaluated the cerebral hemodynamic response to hypercapnia, which is attributable to nonspecific vasodilator action via the relaxation of vascular smooth muscle cells [78]. Baseline CBF was measured under tracheal intubation, immediately before inhalation of $5 \%$ carbon dioxide. Because of the difference of anesthetic drugs, baseline CBF (Fig. 3c; left panels) was lower than resting CBF (Fig. 3a). Once inhalation of 5\% carbon dioxide was started, $\mathrm{CBF}$ rapidly increased in all mice. However, the degree of CBF response to hypercapnia was significantly decreased in vehicle-treated $\mathrm{Tg}$ SwDI mice compared with WT mice (Fig. 3c; upper and middle rows). The CBF response to hypercapnia in taxifolin-treated $\mathrm{Tg}$-SwDI mice was nearly equivalent to that in WT mice (Fig. 3c; lower row and 3d), suggesting taxifolin abolished the impaired CVR in Tg-SwDI mice.

\section{No effects on CBF and CVR by taxifolin treatment in WT mice}

Taxifolin and other flavonols were reported to possess several protective effects on cerebrovasculature $[62,68]$. Taxifolin ameliorated cerebral infarction induced by middle cerebral arterial occlusion through reducing oxidative stress in rats [71]. In order to examine the additive effects of taxifolin on vessels without amyloid- $\beta$ deposits, resting CBF and CBF response to hypercapnia (namely, CVR) was evaluated in taxifolin-treated and vehicle-treated WT mice at 8 months of age. Both mice groups showed comparable levels of resting CBF (Fig. 4a and b) and CVR (Fig. 4c), suggesting that taxifolin did not exert positive effects on intact vessels. 

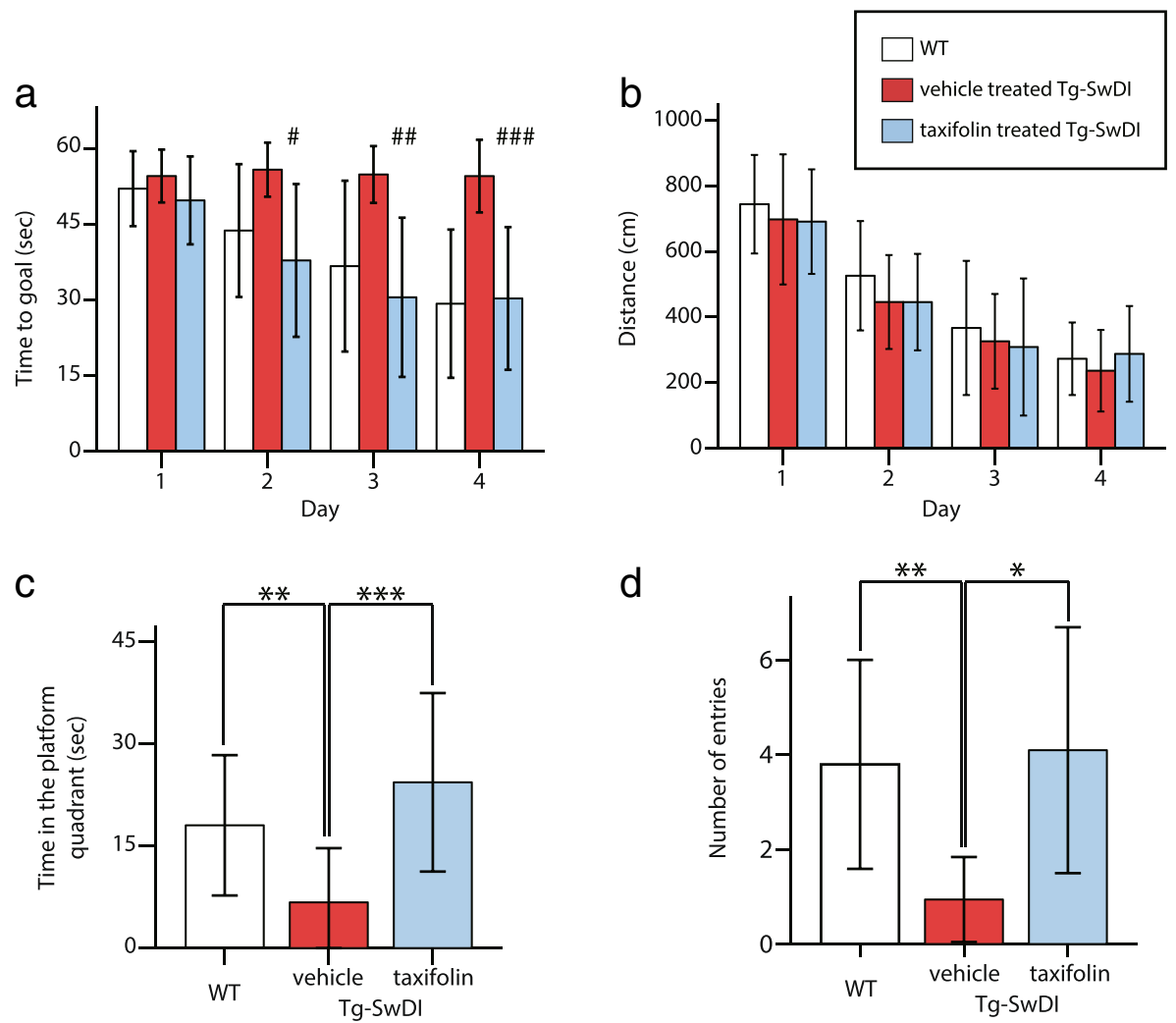

Fig. 2 Effects of taxifolin treatment on cognitive function in the Morris water maze test. Spatial reference memory impairment in Tg-SwDI mice was completely normalized in taxifolin-treated Tg-SwDI mice. (a and $\mathbf{b}$ ) Time course of escape latency (time to goal) (a) and total swimming distance (b) in acquisition phase recorded in vehicle-treated WT (white), vehicle-treated Tg-SwDI (red), and taxifolin-treated Tg-SwDI mice (blue) at 8-months-old receiving taxifolin or vehicle for 7 months ( $n=10-17$ for each group). (c and $\mathbf{d}$ ) The time spent in the target quadrant (c) and the number of entries into target quadrant (d) in the probe trial. Error bars indicate \pm SD. \#p $<0.05$ in WT versus vehicle-treated Tg-SwDI mice and in vehicle-treated versus taxifolin-treated Tg-SwDI mice. \#\#p 0.01 in WT versus vehicle-treated Tg-SwDI mice and in vehicle-treated versus taxifolintreated Tg-SwDI mice. \#\#\#p $<0.001$ in WT versus vehicle-treated Tg-SwDI mice and $p<0.01$ in vehicle-treated versus taxifolin-treated Tg-SwDI mice. ${ }^{*} p<0.05,{ }^{* *} p<0.01,{ }^{* * *} p<0.001$

Decreased vascular amyloid- $\beta$ and amyloid- $\beta_{1-40}$ deposits in taxifolin-treated Tg-SwDI mice

Amyloid- $\beta$ deposits in Tg-SwDI mice were most abundantly observed in the hippocampus (Fig. 5a) and characterized by predominant amyloid- $\beta_{1-40}$ (Fig. 5 b), rather than amyloid- $\beta_{1-42}$ accumulation (Fig. $5 \mathrm{c}$ ). The deposits were distributed in the perivascular areas of cerebral small arteries (Fig. $5 \mathrm{~d}$ and e), which replicated the distribution of amyloid- $\beta$ deposits observed in CAA patients [66].

Immunohistochemical analysis was performed in order to determine whether taxifolin ameliorated cerebrovascular amyloid- $\beta$ accumulation in vivo. Compared with vehicle-treated $\mathrm{Tg}$-SwDI mice, taxifolin-treated Tg-SwDI mice at 8 months of age showed significantly reduced immunoreactivity of both amyloid- $\beta_{1-16}$ (Fig. $5 \mathrm{~g}$ and i) and amyloid- $\beta_{1-40}$ (Fig. 5h and j).

Because cerebral microinfarcts and hemorrhages are commonly observed in CAA patients, we performed H\&E and Perls-Stieda's iron staining as well as immunostaining for glial fibrillary acidic protein and ionized calcium binding adaptor molecule 1: no ischemic or hemorrhagic lesions were observed in taxifolin-treated and vehicletreated Tg-SwDI mice until 12 months of age (Fig. 5f), in accordance with our previous report [51].

\section{No effect on cerebrovascular RAGE expression by taxifolin treatment}

Prior work has demonstrated Silybum marianum suppresses formation of advanced glycation end products (AGEs) [64], and marked anti-glycation activity is a unique property of taxifolin [26]. As RAGE is the major amyloid- $\beta$ influx receptor in the BBB, transporting amyloid- $\beta$ from the blood into the brain, RAGE inhibition was predicted to ameliorate Alzheimer's disease and CAA [14, 15]. We assessed whether taxifolin reduces amyloid- $\beta$ pathology by decreasing RAGE expression in Tg-SwDI mice at 8 months. Cerebrovascular RAGE immunoreactivity was found to be 

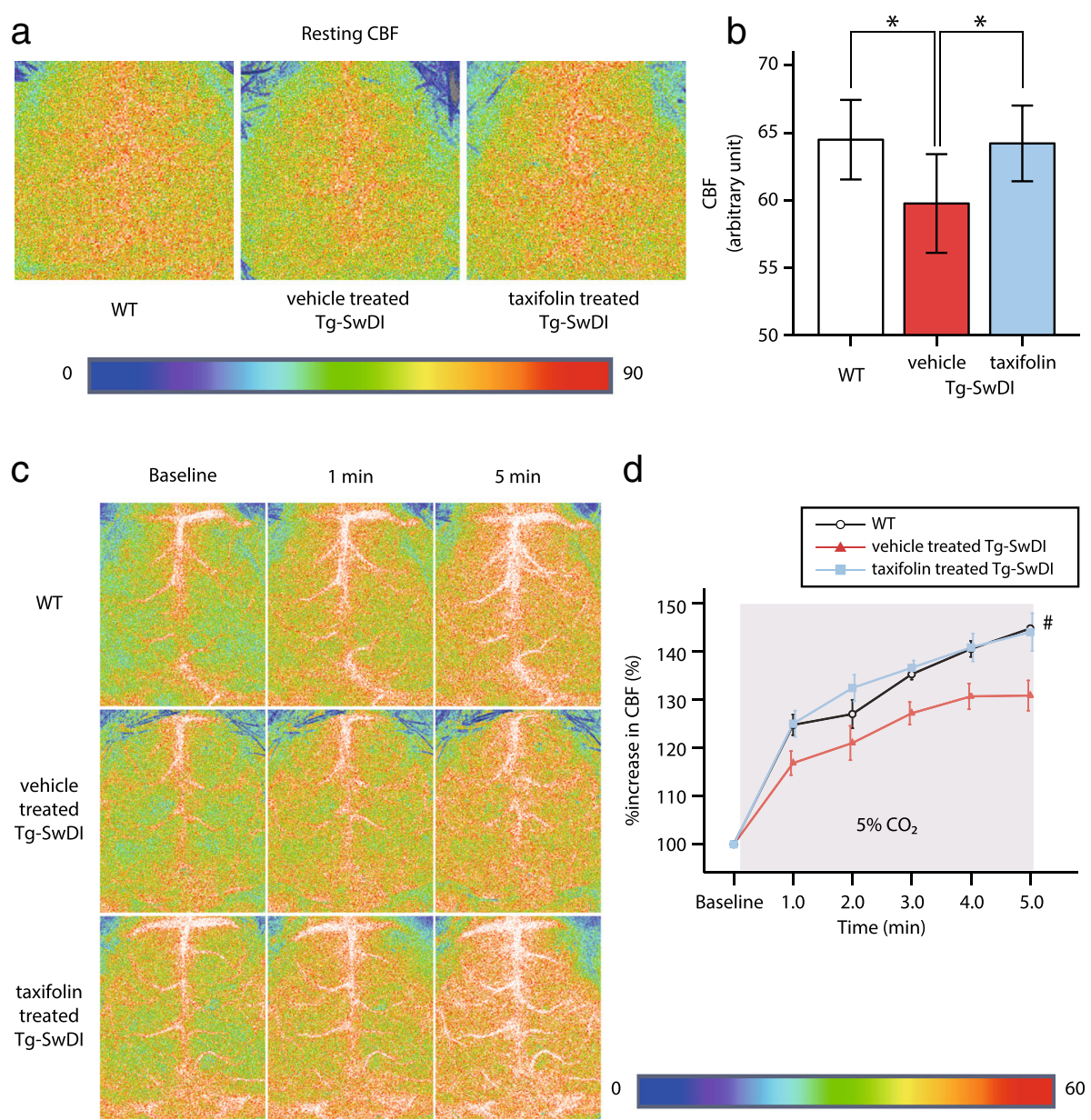

Fig. 3 Effects of taxifolin treatment on CBF and CVR in Tg-SwDI mice. Taxifolin restored CBF reduction and impaired CVR caused by vascular amyloid- $\beta$ in Tg-SwDI mice. a Representative images showing resting CBF measured by laser speckle flowmetry in vehicle-treated WT (left), vehicle-treated (middle), and taxifolin-treated Tg-SwDI (right), mice at 12 months of age receiving taxifolin or vehicle for 11 months. b A histogram showing CBF ( $n=7-13$ for each group). c Representative images of CBF levels monitored by laser speckle flowmetry before (left column; baseline) and 1 min (middle column) and $5 \mathrm{~min}$ (right column) after hypercapnia in vehicle-treated WT (upper row), vehicle-treated (middle row) and taxifolin-treated, Tg-SwDI mice (lower row) at 12 months of age receiving taxifolin or vehicle for 11 months. $\mathbf{d}$ Temporal profiles of \%increase in CBF in WT (black open circle) and Tg-SwDI mice treated with vehicle (red-filled triangle) or taxifolin (blue-filled square) ( $n=4-6$ for each group). Error bars indicate \pm SD (b) and \pm SE (d). ${ }^{*} p<0.05$ and $\# p<0.05$ in WT versus vehicle-treated Tg-SwDI mice and in vehicle-treated versus taxifolin-treated Tg-SwDI mice

comparable between vehicle- and taxifolin-treated groups (Fig. 6a and b).

\section{Amyloid- $\beta_{1-40}$ disassembly by taxifolin treatment}

Preferential accumulation of amyloid- $\beta_{1-40}$ over amyloid$\beta_{1-42}$ is commonly observed in CAA and in Tg-SwDI mice. Therefore, we assessed the effects of taxifolin on amyloid- $\beta_{1-40}$ assembly with an in vitro thioflavin- $T$ fluorescence assay. In the absence of taxifolin, amyloid$\beta_{1-40}$ gradually formed aggregates that bound with thioflavin-T (Fig. 7a). However, the addition of taxifolin decreased thioflavin- $T$ fluorescence intensity, suggesting efficient amyloid- $\beta_{1-40}$ disassembly by taxifolin in vitro. Notably, the addition of $300 \mu \mathrm{M}$ taxifolin resulted in marked amyloid- $\beta_{1-40}$ disassembly. The inhibition of amyloid- $\beta_{1-40}$ fibril formation by taxifolin was also confirmed by TEM imaging (Fig. 7b).

In order to confirm the amyloid- $\beta$ disassembly by taxifolin in vivo, we conducted a filter trap assay and estimated the concentrations of total amyloid- $\beta$ and amyloid- $\beta$ oligomers in TBS soluble fractions of 9-month-old Tg-SwDI mice. Anti-oligomer antibody (A11) binds to the amyloid- $\beta$ oligomer only, while the anti-amyloid- $\beta_{1-16}$ antibody (6E10) detects both the amyloid- $\beta$ monomer and oligomer. The levels of amyloid- $\beta$ oligomers were significantly reduced in taxifolin-treated, compared with vehicle-treated, Tg-SwDI mice (Fig. 7c and e), whereas total soluble amyloid- $\beta$ load was comparable between the two groups (Fig. $7 \mathrm{~d}$ and $\mathrm{f}$ ). 
a Resting CBF

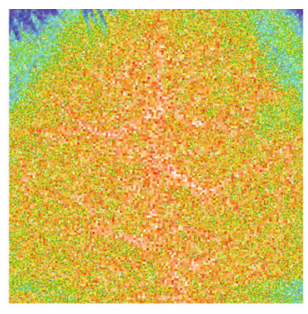

vehicle treated WT

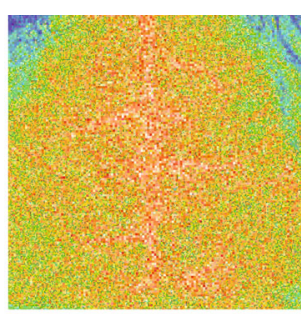

taxifolin treated WT

0

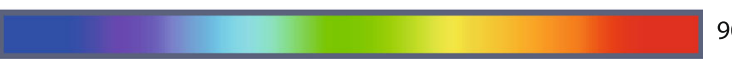

b

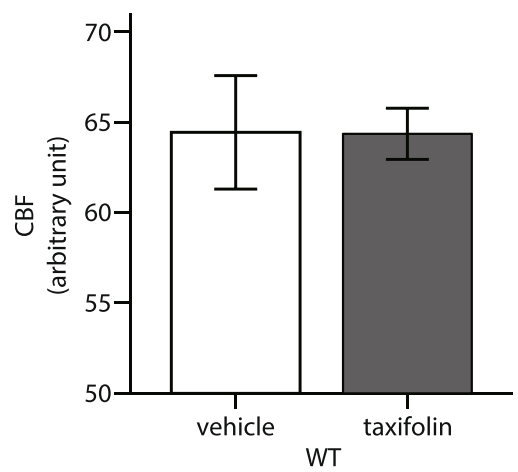

90

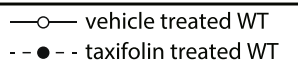

C

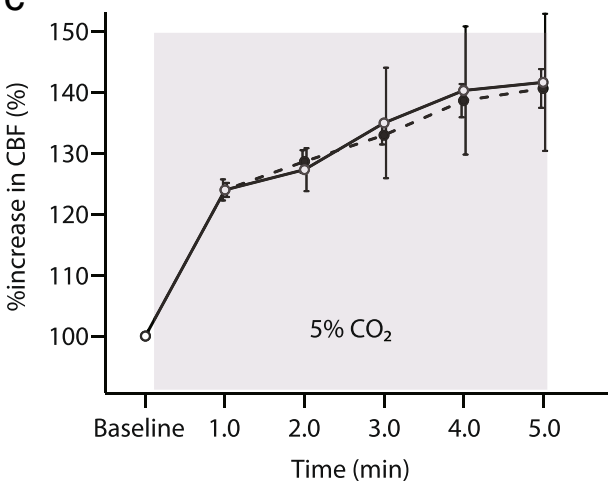

Fig. 4 Effects of taxifolin treatment on CBF and CVR in WT mice. CBF and CVR was not changed between vehicle and taxifolin treated WT mice. a Representative images showing resting CBF measured by laser speckle flowmetry. In vehicle-treated (left), and taxifolin-treated (right), WT mice at 8 months of age receiving vehicle or taxifolin for 7 months. $\mathbf{b}$ A histogram showing CBF ( $n=5-8$ for each). $\mathbf{c}$ Temporal profiles of \%increase in CBF in WT mice aged 8 months with vehicle (open circle) or taxifolin (filled circle) for 7 months ( $n=3$ each). Error bars indicate \pm SD (b) and \pm SE (c)

\section{Amyloid- $\beta$ oligomer disassembly and promotion of amyloid- $\beta$ clearance into blood}

We performed ELISA for amyloid- $\beta_{1-40}$, amyloid- $\beta_{1-42}$, and amyloid- $\beta$ oligomers measurement in TBS soluble and insoluble fraction extracted from 14-month-old TgSwDI mice receiving vehicle or taxifolin. No significant difference was recorded in the concentration of amyloid$\beta_{1-40}$ and amyloid- $\beta_{1-42}$ in TBS soluble fraction between vehicle- and taxifolin-treated groups (Fig. 8a); however, the level of amyloid- $\beta$ oligomers was significantly reduced in taxifolin-treated Tg-SwDI mice, suggesting that taxifolin prevented amyloid- $\beta$ oligomer formation (Fig. 8b). Neither amyloid- $\beta_{1-40}$ nor amyloid- $\beta_{1-42}$ concentration in TBS insoluble fraction was changed by taxifolin treatment (Fig. 8c). The serum level of amyloid$\beta_{1-40}$ level, but not of amyloid- $\beta_{1-42}$, was significantly elevated in 14-month-old Tg-SwDI mice receiving taxifolin compared to vehicle treated $\mathrm{Tg}$-SwDI mice (Fig. 8d). These results indicate that taxifolin suppressed amyloid$\beta$ assembly by inhibiting oligomer formation, which increased amyloid- $\beta$ clearance, preferably amyloid- $\beta_{1-40}$, into the systemic circulation.

\section{Discussion}

Our study revealed taxifolin inhibited amyloid- $\beta_{1-40}$ assembly and oligomer formation, leading to facilitated clearance of amyloid- $\beta$ into the circulation, restoration of CBF and CVR, reduction of amyloid- $\beta$ accumulation and normalization of spatial reference memory impairment in Tg-SwDI, a widely-used CAA mouse model. To our knowledge, this is the first report showing a reduction in the amyloid- $\beta$ oligomer ameliorates the phenotypic expression of CAA, suggesting taxifolin holds potential as a novel therapeutic intervention for CAA.

Taxifolin represents a minor fraction of the flavonols contained in silymarin, typically comprising of approximately $50 \%$ silybin, $20 \%$ silychristin, $10 \%$ silydianin, $5 \%$ isosilybin and an unidentified organic polymer fraction formed from the above compounds [2]. Ability to inhibit amyloid- $\beta_{1-42}$ assembly and to dissolve amyloid- $\beta_{1-42}$ fibrils was reported in taxifolin, but such effects were not observed with silybin, silychristin, and silydianin [60]. Taxifolin is also known to possess multiple pharmacological actions such as anti-oxidation [19], AGE formation suppression [26], and mitochondrial protection [24], and 

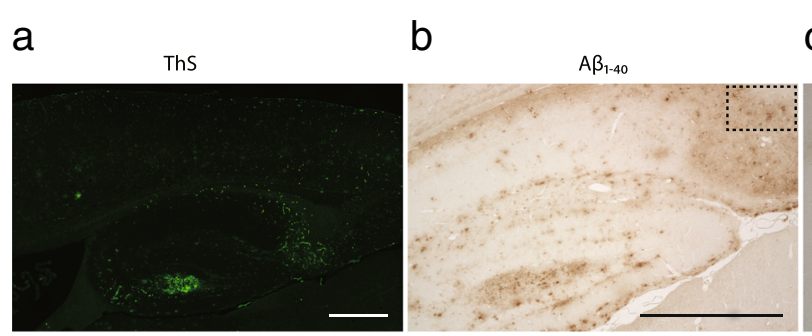

C
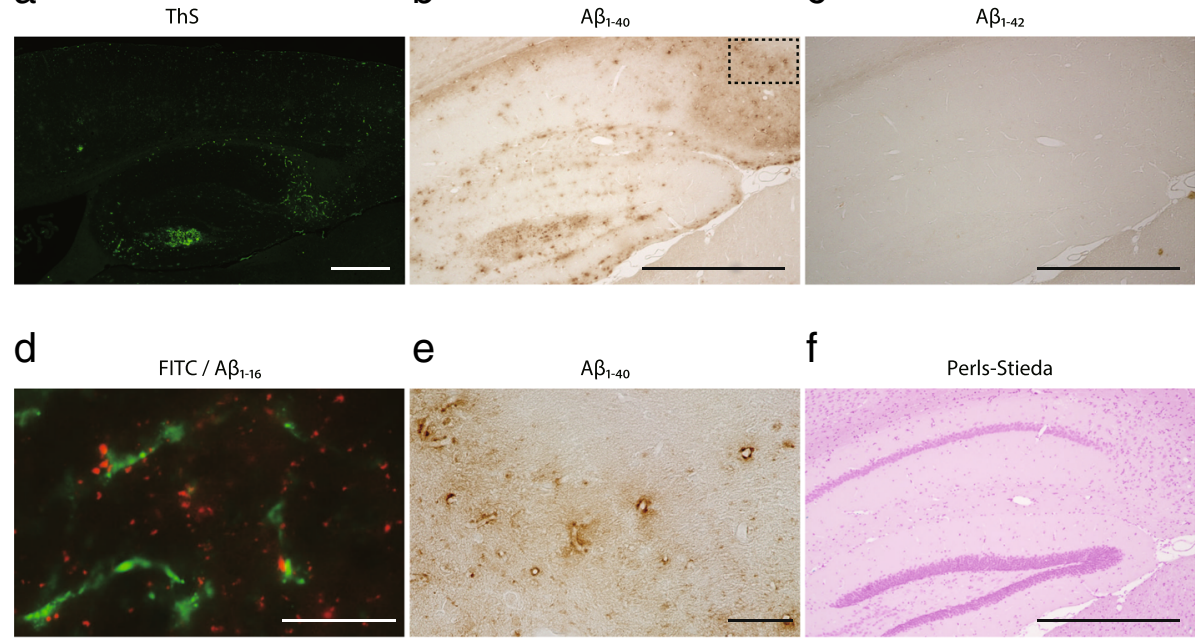

e $\quad A \beta_{1-40}$

f
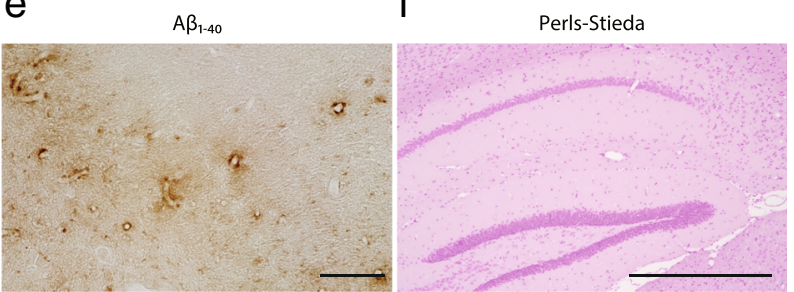

g

h
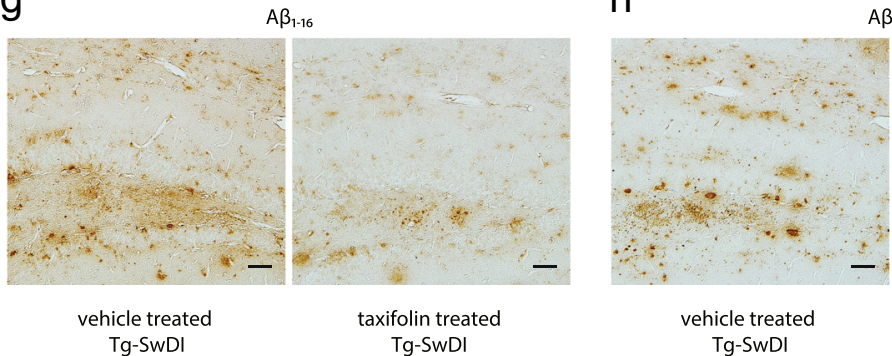

$A \beta_{1-40}$

vehicle treated

Tg-SwDI

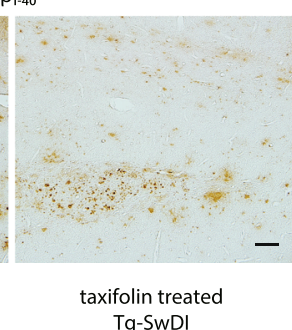

j
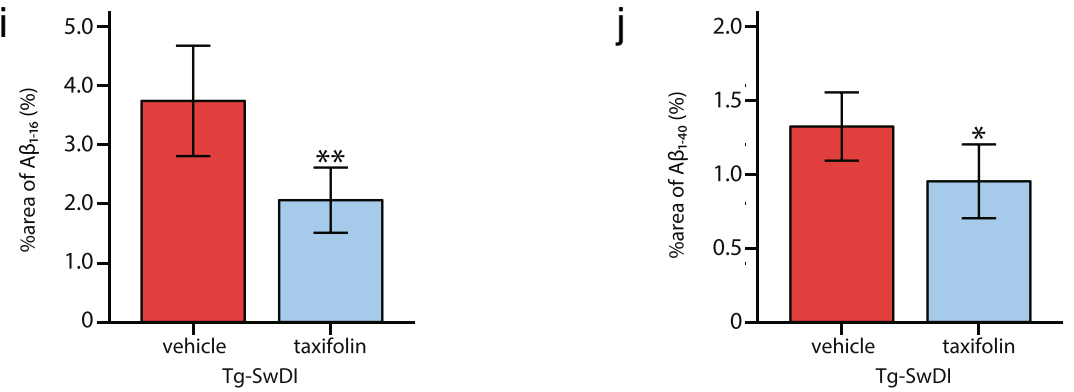

Fig. 5 Effects of taxifolin treatment on amyloid- $\beta$ pathology in Tg-SwDI mice. Vehicle-treated homozygote Tg-SwDI mice exhibited cerebrovascular amyloid- $\beta$ accumulation, which was most abundantly observed in the hippocampus with predominance of amyloid- $\beta_{1-40}$, rather than amyloid- $\beta_{1-42}$ without apparent microhemorrhages or infarcts. Amyloid- $\beta$ deposits were decreased in taxifolin-treated Tg-SwDI mice compared to vehicle-treated $\mathrm{Tg}$-SwDI mice. a Representative image of thioflavin-S staining. Lower magnification of the cortex and the hippocampus in the 12-month-old vehicletreated Tg-SwDI mice. (b, $\mathbf{c}$, e) Representative images of amyloid- $\beta_{1-40}$ and amyloid- $\beta_{1-42}$ immunostaining in the hippocampus in 8-month-old vehicletreated Tg-SwDI mice. $\mathbf{b}$ Lower magnification image of amyloid- $\beta_{1-40}$ immunostaining. c Amyloid- $\beta_{1-42}$ immunostaining. e Higher magnification of amyloid- $\beta_{1-40}$ accumulating vessels (corresponding to dashed square in panel $\mathbf{b}$ ). $\mathbf{d}$ Representative microscopic fluorescent images of amyloid- $\beta_{1-16}$ immunostaining (red) in the hippocampus of the 12-month-old Tg-SwDI mice after FITC dextran (green) injection. Almost all of the amyloid- $\beta_{1-16}$ accumulation is adjacent to cerebral small vessels. $\mathbf{f}$ Representative images of Perls-Stieda's iron staining in the 8-month-old vehicle-treated Tg-SwDI mice. ( $\mathbf{g}$ and $\mathbf{h}$ ) Representative images of amyloid- $\beta_{1-16}(\mathbf{g})$ and amyloid- $\beta_{1-40}(\mathbf{h})$ in the hippocampus of 8-month-old Tg-SwDI mice treated with vehicle (left) or taxifolin (right) for 7 months. (i and $\mathbf{j})$ Histograms showing the density of regions immunoreactive for amyloid- $\beta_{1-16}$ (i) and amyloid- $\beta_{1-}$ 40 (j) in the hippocampus of 8-month-old Tg-SwDI mice treated with vehicle or taxifolin ( $n=5$ each). Scale bars indicate $500 \mu \mathrm{m}(\mathbf{a}, \mathbf{b}, \mathbf{c}, \mathbf{f})$, and $50 \mu \mathrm{m}$ $(\mathbf{d}, \mathbf{e}, \mathbf{g}, \mathbf{h})$. Error bars indicate \pm SD. ${ }^{*} p<0.05$ and ${ }^{* *} p<0.01$

has received increasing attention because of its potential in the treatment of various diseases including malignancy, cardiovascular diseases, chronic hepatitis, hyperlipidemia and neurocognitive disorders [72]. The pharmacokinetic profile of taxifolin has provided essential data, which can be applied to future investigations of various diseases, where such information is lacking. 

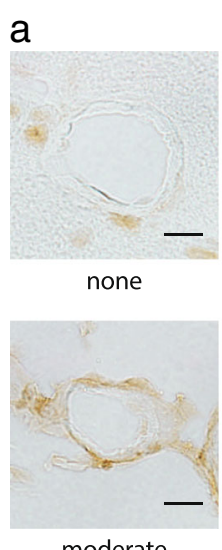

moderate

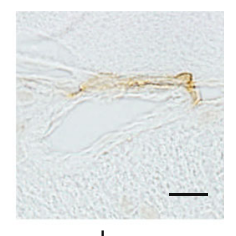

low

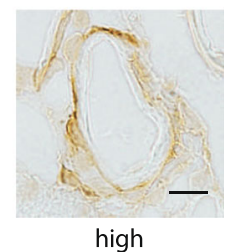

b

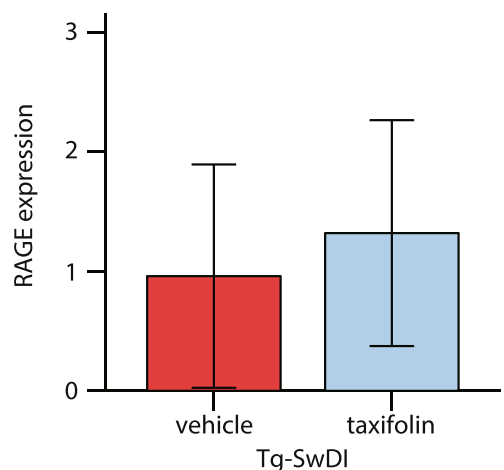

Fig. 6 Effects of taxifolin treatment on cerebrovascular RAGE expression in Tg-SwDI mice. Taxifolin treatment did not significantly change the level of cerebrovascular RAGE expression in Tg-SwDI mice. a Representative images of RAGE immunostaining. $\mathbf{b}$ Histogram showing the grade of cerebrovascular RAGE expression in 8-month-old Tg-SwDI mice receiving vehicle or taxifolin for 7 months. Twenty five leptomeningeal and cortical arteries (five per mouse) for each group. Scale bars indicate $10 \mu \mathrm{m}$. Error bars indicate \pm SD

We detected only small amounts of taxifolin in the brains of both WT and Tg-SwDI mice receiving drug treatment. In order to inhibit both amyloid- $\beta$ production in neural cells and parenchymal amyloid- $\beta$ plaques, drugs need to pass through the BBB, composed of capillary endothelial cells, surrounded by pericytes, basal lamina and the end feet of astrocytic processes [1]. However, drugs aiming for maintenance of cerebrovascular integrity, including taxifolin, need not reach neurons through the BBB [42], providing advantage for clinical application as drug delivery is a frequent problem in the development of novel therapies for neurological diseases.

We administered taxifolin in pelleted chow because of its short elimination half-life. However, the estimated levels of taxifolin were not consistent, and much lower in the daytime than night, suggesting a consistent diurnal concentration of taxifolin is not required to achieve amyloid- $\beta$ disassembly. The low taxifolin concentration in the daytime is likely to result from lower daytime activity and food intake [38]. We did not monitor the timing of food intake, but an earlier report has shown food intake of mice peaks within $6 \mathrm{~h}$ after turning off the light, which was consistent with our pharmacokinetic analysis [77].

The current thioflavin-T assay and TEM analysis showed efficient inhibition of amyloid- $\beta_{1-40}$ assembly. Preventing fibril formation and disentangling preformed fibrils by taxifolin has also been reported in previous investigations $[60,61]$. The decrease in fluorescence intensity in the presented assay is likely to indicate reductions in amyloid- $\beta$ oligomers and preformed fibril dissociation, as oligomers, in addition to amyloid- $\beta$ fibrils, bind to thioflavin $\mathrm{T}$ [41]. The current assay indicated marked amyloid- $\beta_{1-40}$ disassembly when $300 \mu \mathrm{M}$ of taxifolin was added. Taxifolin is known to disassemble amyloid- $\beta$ when auto-oxidated [61]. Oxidation of the catechol moiety of taxifolin results in a formation of oquinone. Oxidized taxifolin is covalently bound to amyloid- $\beta$ at Lys $^{16}$ and Lys $^{28}$ residues, which prevents amyloid- $\beta$ assembly, as Lys ${ }^{16}$ and Lys $^{28}$ residues are critical in $\beta$-sheet formation. Previous reports have shown that amyloid- $\beta$ disassembly with taxifolin proceeds slowly $[60,61]$. Thus, a lesser concentration of taxifolin may disassemble amyloid- $\beta$ if incubated longer. Indeed, amyloid- $\beta_{1-42}$ fibrils can be disentangled by addition of $50 \mu \mathrm{M}$ taxifolin for more than $24 \mathrm{~h}$ [60]. We administered taxifolin to mice from the age of 1 month. Therefore, the long period of administration is likely to contribute to the disassembly of amyloid- $\beta$ by relatively low dose of taxifolin administration compared to the results in vitro.

The pivotal role of amyloid- $\beta$ oligomers in CAA has not been fully clarified, although the pathogenicity of soluble amyloid- $\beta$ in CAA is widely known. Cerebrovascular function was impaired even before the appearance of vascular amyloid- $\beta$ accumulation in Tg-SwDI mice, which were associated with soluble amyloid- $\beta[22,52]$. Passive immunotherapy by ponezumab, intended to mobilize the interstitial fluid pool of amyloid- $\beta$, thus reducing soluble levels, resulted in amelioration of vascular amyloid- $\beta$ accumulation and an improvement of CVR [5]. However, no linkage between CAA severity and amyloid- $\beta$ oligomer expression was documented in a human autopsy study [67]. This may be attributable to limitations of biochemical analysis in human brain samples or bias resulting from the comorbidities of Alzheimer's disease and CAA.

In the present investigation, cerebrovascular dysfunction in Tg-SwDI mice was drastically improved by taxifolin 


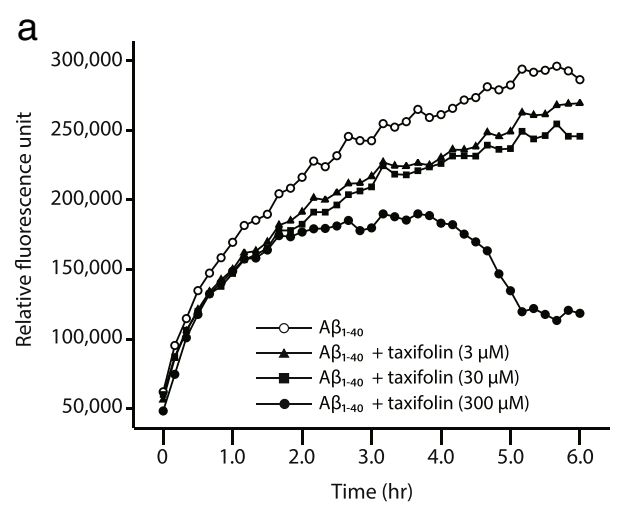

C

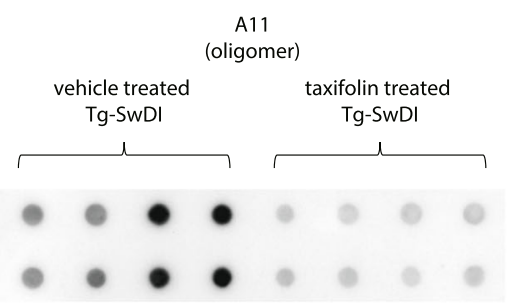

e

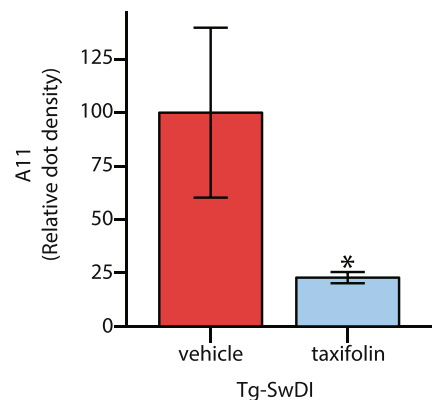

b

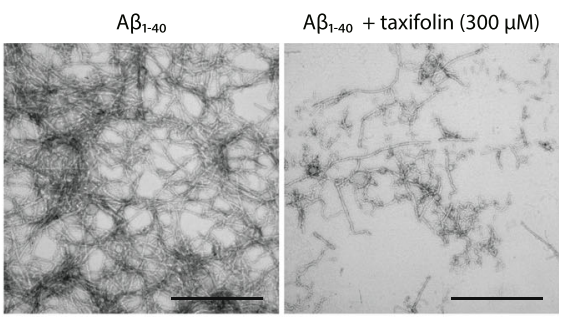

d

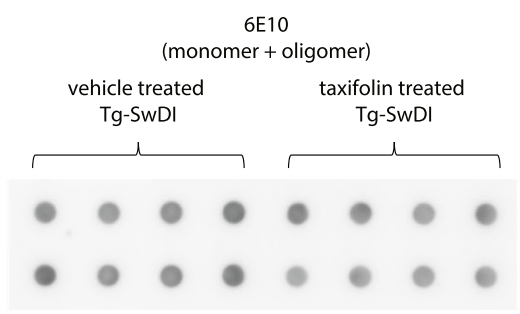

f

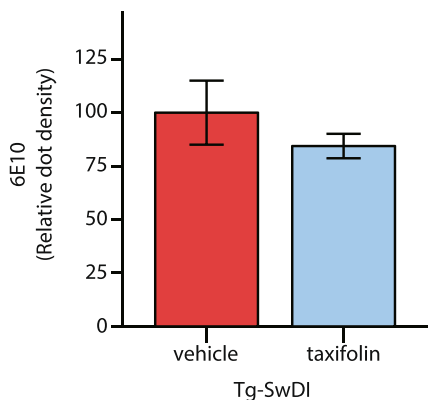

Fig. 7 Effects of taxifolin treatment on amyloid- $\beta$ assembly. Efficient inhibition of amyloid- $\beta_{1-40}$ assembly by taxifolin was shown both in vitro and in vivo. a Amyloid- $\beta_{1-40}$ gradually formed aggregation bound with thioflavin-T in the absence of taxifolin (open circle). However, taxifolin treatment inhibited amyloid- $\beta_{1-40}$ assembly (addition of $3 \mu \mathrm{M}$ of taxifolin: filled triangle, $30 \mu \mathrm{M}$ of taxifolin: filled square, $300 \mu \mathrm{M}$ of taxifolin: filled circle). The mean fluorescence of triply prepared samples was expressed. $\mathbf{b}$ TEM images of fibrils after 6-hour incubation of amyloid- $\beta_{1-40}$ alone (left) and amyloid- $\beta_{1-40}$ with $300 \mu \mathrm{M}$ of taxifolin (right). c-f The results of filter trap assay show taxifolin-treated Tg-SwDI mice have lower levels of amyloid- $\beta$ oligomer than vehicle-treated Tg-SwDI mice. (c and $\mathbf{d}$ ) The blot images of amyloid- $\beta$ in the TBS soluble fractions extracted from the brains of 9-month-old Tg-SwDI mice receiving vehicle or taxifolin for 8 months ( $n=4$ each). The amyloid- $\beta$ oligomers (c) and total amyloid- $\beta$ (d) was detected by anti-amyloid- $\beta$ oligomer antibody (A11) and anti-amyloid- $\beta_{1-16}$ antibody (6E10). The duplicate samples were prepared independently. (e and $\mathbf{f}$ ) Densitometric analysis of amyloid- $\beta$ oligomer (e) and total amyloid- $\beta$ (f). Scale bars indicate $500 \mathrm{~nm}$. Error bars indicate \pm SD. ${ }^{*} p<0.05$

treatment, likely resulting from a reduction of toxic soluble amyloid- $\beta$ species, including oligomers. Since soluble amyloid- $\beta$ has been shown to increase oxidative stress and may result in cerebrovascular deficits [52], strong antioxidant activity of taxifolin may have also contributed to phenotypic recovery in Tg-SwDI mice. However, taxifolin did not change the level of cerebrovascular RAGE expression in Tg-SwDI mice. RAGE is the major amyloid- $\beta$ influx molecule promoting amyloid- $\beta$ transcytosis in the BBB $[14,15]$, suggesting taxifolin was unlikely to affect the AGEs-RAGE axis in Tg-SwDI mice. Taxifolin treatment did not affect CBF and CVR in WT mice, implying taxifolin possesses no additive effects on normal vessels. Thus, the drastic recovery of cognitive and cerebrovascular function shown in taxifolin-treated $\mathrm{Tg}$-SwDI mice could stem mainly from inhibition of amyloid- $\beta$ assembly.

The blood amyloid- $\beta_{1-40}$ level was significantly elevated in taxifolin-treated $\mathrm{Tg}$-SwDI mice. Taxifolin maintained cerebrovascular integrity in Tg-SwDI mice, which might facilitate vascular-mediated removal of amyloid- $\beta$ from the brain [58]. Furthermore, disentangled amyloid- $\beta$ may have been cleared more efficiently through transcytosis across $\mathrm{BBB}$ as well as the perivascular lymphatic drainage (PVD) system [3]. Interstitial fluid and solutes, including 

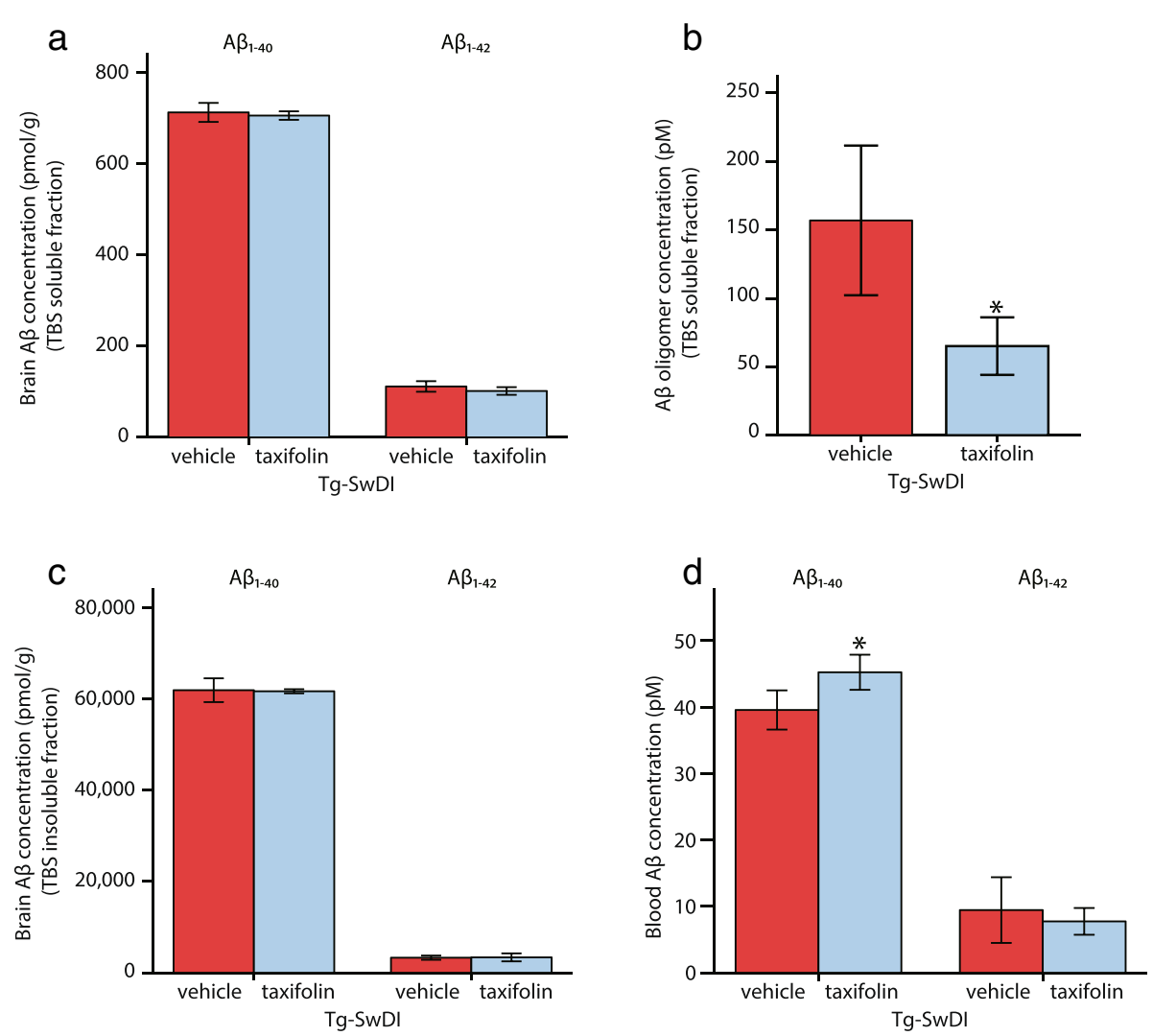

Fig. 8 Effects of taxifolin treatment on oligomer formation and amyloid- $\beta$ clearance. Tg-SwDI mice aged 14 months, which received taxifolin for 13 months, exhibited significant decrease in amyloid- $\beta$ oligomers in the brain and increase of amyloid- $\beta_{1-40}$ clearance into the circulation compared with vehicle-treated Tg-SwDI mice, while the levels of amyloid- $\beta_{1-40}$ and amyloid- $\beta_{1-42}$ in the TBS soluble and insoluble fractions were comparable between vehicle- and taxifolin-treated groups. $\mathbf{a}-\mathbf{d}$ The amount of amyloid- $\beta_{1-40}$, amyloid- $\beta_{1-42}$, and amyloid- $\beta$ oligomers analyzed by ELISA. a The amount of amyloid- $\beta_{1-40}$ and amyloid- $\beta_{1-42}$ in the TBS soluble fraction. $\mathbf{b}$ Amyloid- $\beta$ oligomers in the soluble fraction. $\mathbf{c}$ Amyloid- $\beta_{1-40}$ and amyloid- $\beta_{1-}$ 42 in the insoluble fraction (a-c: $n=4-5$ each). $\mathbf{d}$ The levels of amyloid- $\beta_{1-40}$ and amyloid- $\beta_{1-42}$ in the blood (amyloid- $\beta_{1-40}$ : $n=4-6$ each, amyloid- $\beta_{1-42}$ : $n=3$ each). Error bars indicate \pm SD. ${ }^{*} p<0.05$

amyloid- $\beta$, are cleared from the grey matter through the PVD route, formed by two basement membranes in the walls of cerebral capillaries and arteries, into the cervical lymph nodes and systemic venous systems $[9,45,46]$. A congested PVD pathway could be one of the causes of CAA as amyloid- $\beta$ distribution in CAA closely corresponds with the PVD route [73]. Soluble small molecules have a greater chance of being effectively cleared through the PVD route than large molecules [6]. Thus, increased levels of blood amyloid- $\beta_{1-40}$ in taxifolin-treated Tg-SwDI mice may be attributable to promotion of amyloid- $\beta$ clearance. We reported cilostazol, a selective type-3 phosphodiesterase inhibitor, ameliorated accumulation of amyloid- $\beta$ through facilitation of the PVD route [42]. Synergistic effects may be expected when taxifolin and vasoactive drugs, such as cilostazol, are combined in CAA patients.

Some limitations of our study should be recognized. We analyzed only one strain of CAA model mice. Old- aged Tg2576 and other mice overexpressing APP also exhibited CAA [31], but the use of Tg-SwDI model has two distinctive advantages. Firstly, Tg-SwDI mice exhibit the vasculotropic amyloid- $\beta_{1-40}$ dominant accumulations seen in the human CAA brain [63], although other transgenic models harboring mutant APP genes show abundant amyloid- $\beta_{1-42}$ predominant in parenchymal plaques. Secondly, Tg-SwDI mice express the human transgenic APP at relatively low levels compared to other APP transgenic mice, similar to endogenous mouse APP [12]. Swedish/Dutch/Iowa mutant amyloid- $\beta$ impaired its cerebrovascular-mediated clearance system resulting in robust amyloid- $\beta$ accumulation within cerebral vessels [13]. Since decreased elimination, rather than increased production, of amyloid- $\beta$ is likely to be a major cause of sporadic CAA, as well as Alzheimer's disease [44], this model is useful for investigating mechanisms and therapeutic approaches in CAA $[57,58]$. 
The protective effects of taxifolin against stroke were not confirmed in this study. Cerebral microinfarcts and microbleeds may cause cognitive impairments in patients with $\mathrm{AD}$ and CAA $[11,37]$. Tg-SwDI mice exhibited relatively few cerebral infarcts and bleeds; however, chronic hypoperfusion has been shown to induce cerebral microinfarcts in Tg-SwDI mice [29, 42, 51]. Cerebral microinfarcts are preferentially distributed in arterial borderzone areas in hypoperfused $\mathrm{Tg}$-SwDI mice, and are attributed to impaired CVR. The evaluation of CVR was adopted as a primary endpoint in a phase II clinical trial of ponezumab for probable CAA patients [54]. Since taxifolin fully recovered CVR, taxifolin may help prevent ischemic infarcts in CAA.

\section{Conclusions}

Blocking amyloid- $\beta$ assembly and oligomer formation provides a promising therapeutic target for CAA. Taxifolin maintained cerebrovascular integrity, and completely prevented cognitive decline in CAA model mice. Multifactorial data-driven analysis of information from the Alzheimer's Disease Neuroimaging Initiative uncovered cerebrovascular dysregulation as an early pathological event in the development of Alzheimer's disease [34]. Since the mechanisms behind pathogenesis in Alzheimer's disease and CAA are closely overlapped [59], therapeutic intervention for CAA may also provide a novel diseasemodifying therapy for Alzheimer's disease. A prospective clinical trial is urgently required to determine the effects of taxifolin on CAA and Alzheimer's disease.

\section{Abbreviations}

AGEs: Advanced glycation end products; BBB: Blood-brain barrier;

CAA: Cerebral amyloid angiopathy; CBF: Cerebral blood flow;

Cmax: Maximum concentration; CVR: Cerebrovascular reactivity;

ELISA: Enzyme-linked immunosorbent assay; FITC: Fluorescein isothiocyanate; PVD: Perivascular lymphatic drainage; RAGE: Receptor for advanced glycation end-products; TBS: Tris buffered saline; TEM: Transmission electron microscopy; WT: Wild type

\section{Acknowledgements}

We indebted to Professor Makoto Urushitani and the late Dr. Yoko Okamoto for insightful discussions, as well as Dr. Mikiya Miyazato, Dr. Hiroyuki Kaiya, Dr. Masato Maesako and Dr. Naho Saito for technical advice. We thank to Tetsuya Ishida and Natsuki Hanada for technical assistance and Dr. Ahmad Khundakar for editorial assistance and helpful comments.

\section{Funding}

We gratefully acknowledge grant support from Ministry of Education, Culture, Sports, Science and Technology (M. I., Scientific Research (B), No 15H04271) and Otsuka Pharmaceutical Company.

\section{Authors' contributions}

SS designed the study, performed all the experiments, analyzed and interpreted the data and wrote the manuscript; YY conducted filter-trap assay and wrote the manuscript; TM, YH, MS, RK, RT, supervised and made critical revision of manuscript for important intellectual content; $\mathrm{IH}$ and $\mathrm{KM}$ performed pharmacokinetics testing; MF designed the study, supervised and made critical revision of manuscript for important intellectual content; Ml, designed the study, handled funding, supervised all portions of the study and wrote the manuscript. All authors read and approved the final manuscript.

\section{Competing interests}

Dr. Ito and Mizuno are employees of Otsuka Pharmaceutical Co Ltd. The other authors declare no competing financial interests.

\section{Publisher's Note}

Springer Nature remains neutral with regard to jurisdictional claims in published maps and institutional affiliations.

\section{Author details}

${ }^{1}$ Department of Regenerative Medicine and Tissue Engineering, National Cerebral and Cardiovascular Center, 5-7-1 Fujishiro-dai, Suita, Osaka 565-8565, Japan. ${ }^{2}$ Department of Neurology, Kyoto University Graduate School of Medicine, Kyoto 606-8507, Japan. ${ }^{3}$ Department of Stroke and Cerebrovascular Diseases, National Cerebral and Cardiovascular Center, 5-7-1 Fujishiro-dai, Suita, Osaka 565-8565, Japan. ${ }^{4}$ Department of CNS Research, Otsuka Pharmaceutical Co., Ltd, Tokushima 771-0192, Japan. ${ }^{5}$ Department of Drug Metabolism and Pharmacokinetics, Tokushima Research Institute, Otsuka Pharmaceutical Co., Ltd, Tokushima 771-0192, Japan. 'Department of Molecular Innovation in Lipidology, National Cerebral and Cardiovascular Center, Suita 565-8565, Japan. 'Institute for Ageing and Health, NIHR Biomedical Research Building, Newcastle University, Campus for Ageing and Vitality, Newcastle upon Tyne NE4 5PL, UK. ${ }^{8}$ Department of MediScience, Translational Research Informatics Center, Foundation for Biomedical Research and Innovation, Kobe 650-0047, Japan.

Received: 13 February 2017 Accepted: 22 March 2017

Published online: 04 April 2017

\section{References}

1. Abbott NJ, Ronnback L, Hansson E (2006) Astrocyte-endothelial interactions at the blood-brain barrier. Nat Rev Neurosci 7:41-53. doi:10.1038/nrn1824

2. Abenavoli L, Capasso R, Milic N, Capasso F (2010) Milk thistle in liver diseases: past, present, future. Phytother Res 24:1423-32. doi:10.1002/ptr.3207

3. Arbel-Ornath M, Hudry E, Eikermann-Haerter K, Hou S, Gregory JL, Zhao L, Betensky RA, Frosch MP, Greenberg SM, Bacskai BJ (2013) Interstitial fluid drainage is impaired in ischemic stroke and Alzheimer's disease mouse models. Acta Neuropathol 126:353-64. doi:10.1007/s00401-013-1145-2

4. Ayata C, Dunn AK, Gursoy OY, Huang Z, Boas DA, Moskowitz MA (2004) Laser speckle flowmetry for the study of cerebrovascular physiology in normal and ischemic mouse cortex. J Cereb Blood Flow Metab 24:744-55. doi:10.1097/01.WCB.0000122745.72175.D5

5. Bales KR, O'Neill SM, Pozdnyakov N, Pan F, Caouette D, Pi Y, Wood KM, Volfson D, Cirrito JR, Han BH et al (2016) Passive immunotherapy targeting amyloid- $\beta$ reduces cerebral amyloid angiopathy and improves vascular reactivity. Brain 139:563-77. doi:10.1093/brain/awv313

6. Barua NU, Bienemann AS, Hesketh S, Wyatt MJ, Castrique E, Love S, Gill SS (2012) Intrastriatal convection-enhanced delivery results in widespread perivascular distribution in a pre-clinical model. Fluids Barriers CNS 9:2. doi: 10.1186/2045-8118-9-2

7. Booth AN, Deeds F (1958) The toxicity and metabolism of dihydroquercetin. J Am Pharm Assoc Am Pharm Assoc 47:183-4

8. Boyle PA, Yu L, Nag S, Leurgans S, Wilson RS, Bennett DA, Schneider JA (2015) Cerebral amyloid angiopathy and cognitive outcomes in community-based older persons. Neurology 85:1930-6. doi:10.1212/WNL.0000000000002175

9. Carare RO, Bernardes-Silva M, Newman TA, Page AM, Nicoll JA, Perry VH, Weller RO (2008) Solutes, but not cells, drain from the brain parenchyma along basement membranes of capillaries and arteries: significance for cerebral amyloid angiopathy and neuroimmunology. Neuropathol Appl Neurobiol 34:131-44. doi:10.1111/j.1365-2990.2007.00926.x

10. Case NF, Charlton A, Zwiers A, Batool S, McCreary CR, Hogan DB, Ismail Z, Zerna C, Coutts SB, Frayne R et al (2016) Cerebral amyloid angiopathy is associated with executive dysfunction and mild cognitive impairment. Stroke 47:2010-6. doi:10.1161/STROKEAHA.116.012999

11. Chiang GC, Cruz Hernandez JC, Kantarci K, Jack CR Jr, Weiner MW (2015) Cerebral microbleeds, CSF p-Tau, and cognitive decline: significance of anatomic distribution. AJNR Am J Neuroradiol 36:1635-41. doi:10.3174/ajnr.A4351

12. Davis J, Xu F, Deane R, Romanov G, Previti ML, Zeigler K, Zlokovic BV, Van Nostrand WE (2004) Early-onset and robust cerebral microvascular accumulation of amyloid $\beta$-protein in transgenic mice expressing low levels of a vasculotropic Dutch/lowa mutant form of amyloid $\beta$-protein precursor. J Biol Chem 279:20296-306. doi:10.1074/jbc.M312946200 
13. Davis J, Xu F, Miao J, Previti ML, Romanov G, Ziegler K, Van Nostrand WE (2006) Deficient cerebral clearance of vasculotropic mutant Dutch/lowa Double A $\beta$ in human AßPP transgenic mice. Neurobiol Aging 27:946-54. doi:10.1016/j.neurobiolaging.2005.05.031

14. Deane R, Du Yan S, Submamaryan RK, LaRue B, Jovanovic S, Hogg E, Welch D, Manness L, Lin C, Yu J et al (2003) RAGE mediates amyloid- $\beta$ peptide transport across the blood-brain barrier and accumulation in brain. Nat Med 9:907-13. doi:10.1038/nm890

15. Deane R, Singh I, Sagare AP, Bell RD, Ross NT, LaRue B, Love R, Perry S, Paquette N, Deane RJ et al (2012) A multimodal RAGE-specific inhibitor reduces amyloid $\beta$-mediated brain disorder in a mouse model of Alzheimer disease. J Clin Invest 122:1377-92. doi:10.1172/JCI58642

16. Dumas A, Dierksen GA, Gurol ME, Halpin A, Martinez-Ramirez S, Schwab K, Rosand J, Viswanathan A, Salat DH, Polimeni JR et al (2012) Functional magnetic resonance imaging detection of vascular reactivity in cerebral amyloid angiopathy. Ann Neurol 72:76-81. doi:10.1002/ana.23566

17. Glenner GG, Wong CW (1984) Alzheimer's disease: initial report of the purification and characterization of a novel cerebrovascular amyloid protein. Biochem Biophys Res Commun 120:885-90

18. Greenberg SM, Al-Shahi Salman R, Biessels GJ, van Buchem M, Cordonnier C, Lee JM, Montaner J, Schneider JA, Smith EE, Vernooij M et al (2014) Outcome markers for clinical trials in cerebral amyloid angiopathy. Lancet Neurol 13:419-28. doi:10.1016/S1474-4422(14)70003-1

19. Guo H, Zhang X, Cui Y, Zhou H, Xu D, Shan T, Zhang F, Guo Y, Chen Y, Wu D (2015) Taxifolin protects against cardiac hypertrophy and fibrosis during biomechanical stress of pressure overload. Toxicol Appl Pharmacol 287:168-77. doi:10.1016/j.taap.2015.06.002

20. Gupta A, ladecola C (2015) Impaired A atherosclerosis and Alzheimer's disease. Front Aging Neurosci 7:115. doi:10.3389/fnagi.2015.00115

21. Haass C, Selkoe DJ (2007) Soluble protein oligomers in neurodegeneration: lessons from the Alzheimer's amyloid $\beta$-peptide. Nat Rev Mol Cell Biol 8 : 101-12. doi:10.1038/nrm2101

22. Han BH, Zhou ML, Abousaleh F, Brendza RP, Dietrich HH, Koenigsknecht Talboo J, Cirrito JR, Milner E, Holtzman DM, Zipfel GJ (2008) Cerebrovascular dysfunction in amyloid precursor protein transgenic mice: contribution of soluble and insoluble amyloid- $\beta$ peptide, partial restoration via $\gamma$-secretase inhibition. J Neurosci 28:13542-50. doi:10.1523/JNEUROSCI.4686-08.2008

23. Han BH, Zhou ML, Johnson AW, Singh I, Liao F, Vellimana AK, Nelson JW, Milner E, Cirrito JR, Basak J et al (2015) Contribution of reactive oxygen species to cerebral amyloid angiopathy, vasomotor dysfunction, and microhemorrhage in aged Tg2576 mice. Proc Natl Acad Sci U S A 112:E881-E90. doi:10.1073/pnas.1414930112

24. Haraguchi H, Mochida Y, Sakai S, Masuda H, Tamura Y, Mizutani K, Tanaka O, Chou WH (1996) Protection against oxidative damage by dihydroflavonols in Engelhardtia chrysolepis. Biosci Biotechnol Biochem 60:945-8. doi:10.1271/bbb.60.945

25. Hardy JA, Higgins GA (1992) Alzheimer's disease: the amyloid cascade hypothesis. Science 256:184-5

26. Harris CS, Beaulieu LP, Fraser MH, McIntyre KL, Owen PL, Martineau LC, Cuerrier A, Johns T, Haddad PS, Bennett SA et al (2011) Inhibition of advanced glycation end product formation by medicinal plant extracts correlates with phenolic metabolites and antioxidant activity. Planta Med 77:196-204. doi:10.1055/s-0030-1250161

27. Hattori Y, Enmi J, Iguchi S, Saito S, Yamamoto Y, Tsuji M, Nagatsuka K, Kalaria RN, lida H, Ihara M (2016) Gradual carotid artery stenosis in mice closely replicates hypoperfusive vascular dementia in humans. J Am Heart Assoc 5:e002757. doi:10.1161/JAHA.115.002757

28. Hattori Y, Enmi J, Kitamura A, Yamamoto Y, Saito S, Takahashi Y, Iguchi S, Tsuji M, Yamahara K, Nagatsuka K et al (2015) A novel mouse model of subcortical infarcts with dementia. J Neurosci 35:3915-28. doi:10.1523/ JNEUROSCI.3970-14.2015

29. Hattori Y, Maki T, Saito S, Yamamoto Y, Nagatsuka K, Ihara M (2016) Influence of Low-dose aspirin on cerebral amyloid angiopathy in mice. J Alzheimers Dis 52:1037-45. doi:10.3233/JAD-160013

30. Hattori Y, Okamoto Y, Maki T, Yamamoto Y, Oishi N, Yamahara K, Nagatsuka K, Takahashi R, Kalaria RN, Fukuyama H et al (2014) Silent information regulator 2 homolog 1 counters cerebral hypoperfusion injury by deacetylating endothelia nitric oxide synthase. Stroke 45:3403-11. doi:10.1161/STROKEAHA.114.006265

31. Hawkes CA, Hartig W, Kacza J, Schliebs R, Weller RO, Nicoll JA, Carare RO (2011) Perivascular drainage of solutes is impaired in the ageing mouse brain and in the presence of cerebral amyloid angiopathy. Acta Neuropathol 121:431-43. doi:10.1007/s00401-011-0801-7

32. Hellstrand E, Boland B, Walsh DM, Linse S (2010) Amyloid $\beta$-protein aggregation produces highly reproducible kinetic data and occurs by a two-phase process. ACS Chem Neurosci 1:13-8. doi:10.1021/cn900015V

33. Hudson SA, Ecroyd H, Kee TW, Carver JA (2009) The thioflavin T fluorescence assay for amyloid fibril detection can be biased by the presence of exogenous compounds. FEBS J 276:5960-72. doi:10.1111/j.1742-4658.2009.07307.x

34. Iturria-Medina Y, Sotero RC, Toussaint PJ, Mateos-Perez JM, Evans AC (2016) Alzheimer's disease neuroimaging I. Early role of vascular dysregulation on ate-onset Alzheimer's disease based on multifactorial data-driven analysis. Nat Commun 7:11934. doi:10.1038/ncomms11934

35. Kalaria RN (2016) Neuropathological diagnosis of vascular cognitive impairment and vascular dementia with implications for Alzheimer's disease. Acta Neuropathol 131:659-85. doi:10.1007/s00401-016-1571-z

36. Keage HA, Carare RO, Friedland RP, Ince PG, Love S, Nicoll JA, Wharton SB, Weller RO, Brayne C (2009) Population studies of sporadic cerebral amyloid angiopathy and dementia: a systematic review. BMC Neurol 9:3. doi:10. 1186/1471-2377-9-3

37. Launer LJ, Hughes TM, White LR (2011) Microinfarcts, brain atrophy, and cognitive function: the Honolulu Asia aging study autopsy study. Ann Neurol 70:774-80. doi:10.1002/ana.22520

38. LeGates TA, Altimus CM. Measuring circadian and acute light responses in mice using wheel running activity. J Vis Exp. 2011:e2463. doi:10.3791/2463.

39. Maesako M, Uemura K, Iwata A, Kubota M, Watanabe $K$, Uemura M, Noda $Y$, Asada-Utsugi M, Kihara T, Takahashi R et al (2013) Continuation of exercise is necessary to inhibit high fat diet-induced $\beta$-amyloid deposition and memory deficit in amyloid precursor protein transgenic mice. PLoS One 8: e72796. doi:10.1371/journal.pone.0072796

40. Maesako M, Uemura K, Kubota M, Kuzuya A, Sasaki K, Hayashida N, AsadaUtsugi M, Watanabe K, Uemura M, Kihara T et al (2012) Exercise is more effective than diet control in preventing high fat diet-induced $\beta$-amyloid deposition and memory deficit in amyloid precursor protein transgenic mice. J Biol Chem 287:23024-33. doi:10.1074/jbc.M112.367011

41. Maezawa I, Hong HS, Liu R, Wu CY, Cheng RH, Kung MP, Kung HF, Lam KS, Oddo S, Laferla FM et al (2008) Congo red and thioflavin-T analogs detect A $\beta$ oligomers. J Neurochem 104:457-68. doi:10.1111/j.1471-4159.2007.04972.x

42. Maki $T$, Okamoto $Y$, Carare RO, Hase $Y$, Hattori $Y$, Hawkes CA, Saito $S$, Yamamoto Y, Terasaki Y, Ishibashi-Ueda H et al (2014) Phosphodiesterase III inhibitor promotes drainage of cerebrovascular $\beta$-amyloid. Ann Clin Transl Neurol 1:519-33. doi:10.1002/acn3.79

43. Masuda Y, Nakanishi A, Ohashi R, Takegoshi K, Shimizu T, Shirasawa T, Irie K (2008) Verification of the intermolecular parallel $\beta$-sheet in E22K-Aß42 aggregates by solid-state NMR using rotational resonance: implications for the supramolecular arrangement of the toxic conformer of Aß42. Biosci Biotechnol Biochem 72:2170-5. doi:10.1271/bbb.80250

44. Mawuenyega KG, Sigurdson W, Ovod V, Munsell L, Kasten T, Morris JC, Yarasheski KE, Bateman RJ (2010) Decreased clearance of CNS $\beta$-amyloid in Alzheimer's disease. Science 330:1774. doi:10.1126/science.1197623

45. Morris AWJ, Carare RO, Schreiber S, Hawkes CA (2014) The cerebrovascular basement membrane: role in the clearance of $\beta$ amyloid and cerebral amyloid angiopathy. Front Aging Neurosci 6:251. doi:10.3389/fnagi.2014.00251

46. Morris AWJ, Sharp MM, Albargothy NJ, Fernandes R, Hawkes CA, Verma A, Weller RO, Carare RO (2016) Vascular basement membranes as pathways for the passage of fluid into and out of the brain. Acta Neuropathol 131:725-36. doi:10.1007/s00401-016-1555-z

47. Moulin S, Labreuche J, Bombois S, Rossi C, Boulouis G, Henon H, Duhamel A, Leys D, Cordonnier C (2016) Dementia risk after spontaneous intracerebral haemorrhage: a prospective cohort study. Lancet Neurol 15: 820-9. doi:10.1016/S1474-4422(16)00130-7

48. Murata N, Murakami K, Ozawa Y, Kinoshita N, Irie K, Shirasawa T, Shimizu T (2010) Silymarin attenuated the amyloid $\beta$ plaque burden and improved behavioral abnormalities in an Alzheimer's disease mouse model. Biosci Biotechnol Biochem 74:2299-306. doi:10.1271/bbb.100524

49. Niwa K, Carlson GA, ladecola C (2000) Exogenous Aß1-40 reproduces cerebrovascular alterations resulting from amyloid precursor protein overexpression in mice. J Cereb Blood Flow Metab 20:1659-68. doi:10.1097/ 00004647-200012000-00005

50. Niwa K, Younkin L, Ebeling C, Turner SK, Westaway D, Younkin S, Ashe KH, Carlson GA, ladecola C (2000) AB1-40-related reduction in functional 
hyperemia in mouse neocortex during somatosensory activation. Proc Nat Acad Sci U S A 97:9735-40

51. Okamoto Y, Yamamoto T, Kalaria RN, Senzaki H, Maki T, Hase Y, Kitamura A, Washida K, Yamada M, Ito H et al (2012) Cerebral hypoperfusion accelerates cerebral amyloid angiopathy and promotes cortical microinfarcts. Acta Neuropathol 123:381-94. doi:10.1007/s00401-011-0925-9

52. Park L, Koizumi K, El Jamal S, Zhou P, Previti ML, Van Nostrand WE, Carlson G, ladecola C (2014) Age-dependent neurovascular dysfunction and damage in a mouse model of cerebral amyloid angiopathy. Stroke 45:1815-21. doi:10.1161/STROKEAHA.114.005179

53. Park L, Zhou P, Koizumi K, El Jamal S, Previti ML, Van Nostrand WE, Carlson G, ladecola C (2013) Brain and circulating levels of A 1-40 differentially contribute to vasomotor dysfunction in the mouse brain. Stroke 44:198-204. doi:10.1161/STROKEAHA.112.670976

54. Pfizer. Study Evaluating the Safety,Tolerability and Efficacy of PF-04360365 in Adults With Probable Cerebral Amyloid Angiopathy. In: Clinical Trials.gov. The U.S. National Institutes of Health. 2016. https:/clinicaltrials.gov/ct2/show/study/ NCT01821118?term=ponezumab. Accessed 15 Jan 2017.

55. Reijmer YD, Fotiadis P, Martinez-Ramirez S, Salat DH, Schultz A, Shoamanesh A, Ayres AM, Vashkevich A, Rosas D, Schwab K et al (2015) Structural network alterations and neurological dysfunction in cerebral amyloid angiopathy. Brain 138:179-88. doi:10.1093/brain/awu316

56. Rovelet-Lecrux A, Hannequin D, Raux G, Le Meur N, Laquerriere A, Vital A, Dumanchin C, Feuillette S, Brice A, Vercelletto M et al (2006) APP locus duplication causes autosomal dominant early-onset Alzheimer disease with cerebral amyloid angiopathy. Nat Genet 38:24-6. doi:10.1038/ng1718

57. Saito S, Ihara M (2016) Interaction between cerebrovascular disease and Alzheimer pathology. Curr Opin Psychiatry 29:168-73. doi:10.1097/YCO. 0000000000000239

58. Saito S, Ihara M (2014) New therapeutic approaches for Alzheimer's disease and cerebral amyloid angiopathy. Front Aging Neurosci 6:290. doi:10.3389/ fnagi.2014.00290

59. Saito S, Yamamoto Y, Ihara M (2015) Mild Cognitive Impairment: At the Crossroad of Neurodegeneration and Vascular Dysfunction. Curr Alzheimer Res 12:507-12. doi:10.2174/1567205012666150530202508

60. Sato M, Murakami K, Uno M, Ikubo H, Nakagawa Y, Katayama S, Akagi K, Irie K (2013) Structure-activity relationship for (+)-taxifolin isolated from silymarin as an inhibitor of amyloid $\beta$ aggregation. Biosci Biotechnol Biochem 77:1100-3. doi:10.1271/bbb.120925

61. Sato M, Murakami K, Uno M, Nakagawa Y, Katayama S, Akagi K, Masuda Y, Takegoshi K, Irie K (2013) Site-specific inhibitory mechanism for amyloid $\beta 42$ aggregation by catechol-type flavonoids targeting the Lys residues. J Biol Chem 288:23212-24. doi:10.1074/jbc.M113.464222

62. Schroeter H, Heiss C, Balzer J, Kleinbongard P, Keen CL, Hollenberg NK, Sies H, Kwik-Uribe C, Schmitz HH, Kelm M (2006) (-)-Epicatechin mediates beneficial effects of flavanol-rich cocoa on vascular function in humans. Proc Natl Acad Sci U S A 103:1024-9. doi:10.1073/pnas.0510168103

63. Searcy JL, Le Bihan T, Salvadores N, McCulloch J, Horsburgh K (2014) Impact of age on the cerebrovascular proteomes of wild-type and Tg-SwDI mice. PLoS One 9:e89970. doi:10.1371/journal.pone.0089970

64. Shin S, Lee JA, Kim M, Kum H, Jung E, Park D (2015) Anti-glycation activities of phenolic constituents from Silybum marianum (Milk Thistle) flower in vitro and on human explants. Molecules 20:3549-64. doi:10.3390/ molecules 20033549

65. Smith EE, Greenberg SM (2009) $\beta$-amyloid, blood vessels, and brain function. Stroke 40:2601-6. doi:10.1161/STROKEAHA.108.536839

66. Thal DR, Griffin WS, de Vos RA, Ghebremedhin E (2008) Cerebral amyloid angiopathy and its relationship to Alzheimer's disease. Acta Neuropathol 115:599-609. doi:10.1007/s00401-008-0366-2

67. van Helmond Z, Miners JS, Kehoe PG, Love S (2010) Oligomeric A $\beta$ in Alzheimer's disease: relationship to plaque and tangle pathology, APOE genotype and cerebral amyloid angiopathy. Brain Pathol 20:468-80. doi:10.1111/j.1750-3639.2009.00321.x

68. Vauzour D, Vafeiadou K, Rodriguez-Mateos A, Rendeiro C, Spencer JP (2008) The neuroprotective potential of flavonoids: a multiplicity of effects. Genes Nutr 3:115-26. doi:10.1007/s12263-008-0091-4

69. Viswanathan A, Greenberg SM (2011) Cerebral amyloid angiopathy in the elderly. Ann Neurol 70:871-80. doi:10.1002/ana.22516

70. Wang X, Xia H, Xing F, Deng G, Shen Q, Zeng S (2009) A highly sensitive and robust UPLC-MS with electrospray ionization method for quantitation of taxifolin in rat plasma. J Chromatogr B Analyt Technol Biomed Life Sci 877:1778-86. doi:10.1016/j.jchromb.2009.04.037

71. Wang YH, Wang WY, Chang CC, Liou KT, Sung YJ, Liao JF, Chen CF, Chang S, Hou YC, Chou YC et al (2006) Taxifolin ameliorates cerebral ischemiareperfusion injury in rats through its anti-oxidative effect and modulation of NF-kappa B activation. J Biomed Sci 13:127-41. doi:10.1007/s11373-005-9031-0

72. Weidmann AE (2012) Dihydroquercetin: more than just an impurity? Eur J Pharmacol 684:19-26. doi:10.1016/j.ejphar.2012.03.035

73. Weller RO, Hawkes CA, Carare RO, Hardy J (2015) Does the difference between PART and Alzheimer's disease lie in the age-related changes in cerebral arteries that trigger the accumulation of $A B$ and propagation of tau? Acta Neuropathol 129:763-6. doi:10.1007/s00401-015-1416-1

74. Wiseman FK, Al-Janabi T, Hardy J, Karmiloff-Smith A, Nizetic D, Tybulewicz VL, Fisher EM, Strydom A (2015) A genetic cause of Alzheimer disease: mechanistic insights from down syndrome. Nat Rev Neurosci 16:564-74. doi:10.1038/nrn3983

75. Yamada M, Ihara M, Okamoto Y, Maki T, Washida K, Kitamura A, Hase Y, Ito H, Takao K, Miyakawa T et al (2011) The influence of chronic cerebral hypoperfusion on cognitive function and amyloid $\beta$ metabolism in APP overexpressing mice. PLoS One 6:e16567. doi:10.1371/journal.pone.0016567

76. Yang P, Xu F, Li HF, Wang Y, Li FC, Shang MY, Liu GX, Wang X, Cai SQ. Detection of 191 Taxifolin Metabolites and Their Distribution in Rats Using HPLC-ESI-IT-TOF-MS ${ }^{n}$. Molecules. 2016;21. doi:10.3390/molecules21091209

77. Yasumoto Y, Nakao R, Oishi K (2015) Free access to a running-wheel advances the phase of behavioral and physiological circadian rhythms and peripheral molecular clocks in mice. PLoS One 10:e0116476. doi:10.1371/ journal.pone.0116476

78. Yoon S, Zuccarello M, Rapoport RM (2012) $\mathrm{pCO}_{2}$ and $\mathrm{pH}$ regulation of cerebral blood flow. Front Physiol 3:365. doi:10.3389/fphys.2012.00365

79. Zhanataev AK, Kulakova AV, Nasonova W, Durnev AD (2008) In vivo study of dihydroquercetin genotoxicity. Bull Exp Biol Med 145:338-40

\section{Submit your next manuscript to BioMed Central and we will help you at every step:}

- We accept pre-submission inquiries

- Our selector tool helps you to find the most relevant journal

- We provide round the clock customer support

- Convenient online submission

- Thorough peer review

- Inclusion in PubMed and all major indexing services

- Maximum visibility for your research

Submit your manuscript at www.biomedcentral.com/submit
Ciomed Central 